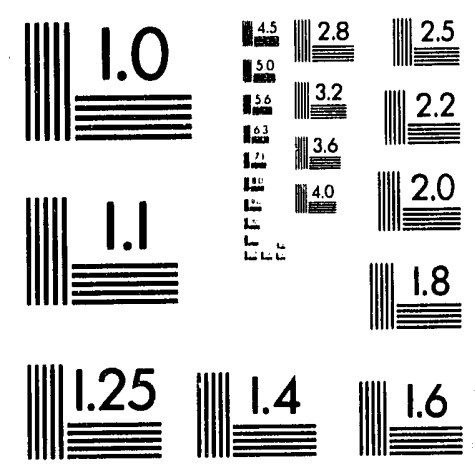



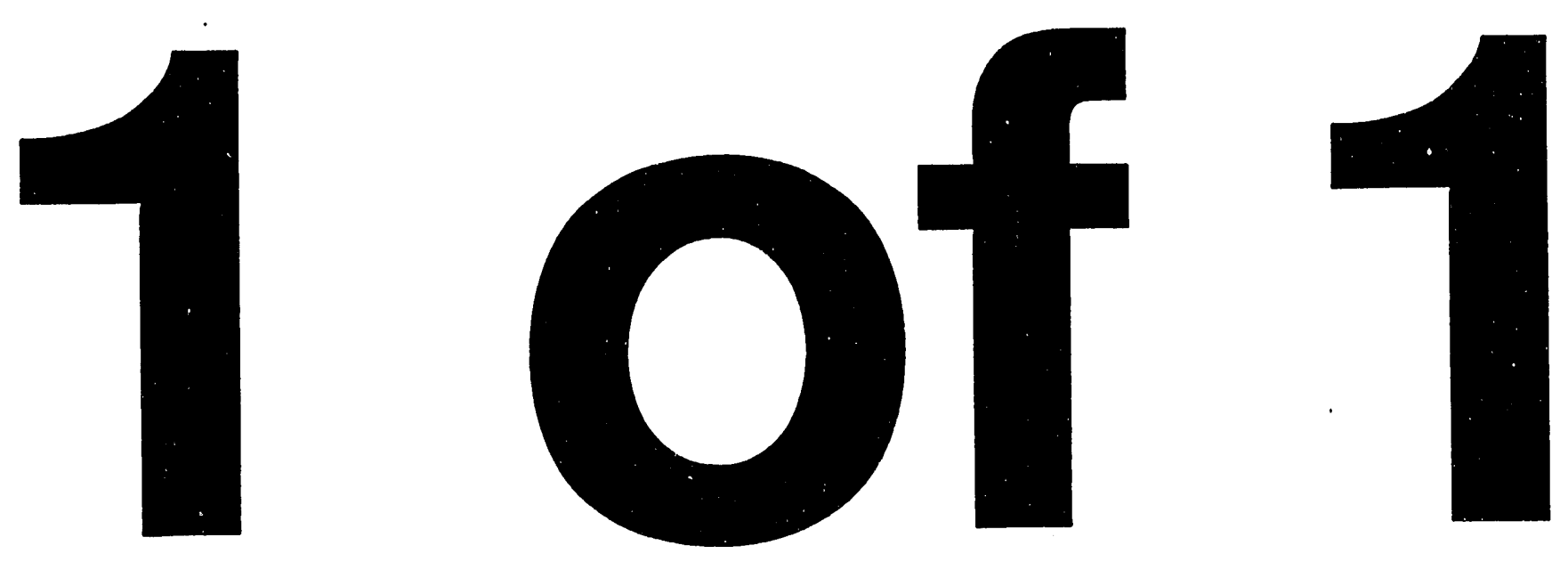
PREPARED FOR THE U.S. DEPARTMENT OF ENERGY, UNDER CONTRACT DE-AC02-76-CHO-3073

PPPL-2935

UC-427

PPPL-2935

\section{THEORY OF BALLOONING-MIRROR INSTABILITIES FOR ANISOTROPIC PRESSURE PLASMAS IN THE MAGNETOSPHERE}

BY

C.Z. CHENG AND Q. QIAN

SEPTEMBER, 1993

$\int \frac{D}{5} \int 5 \int\left[\begin{array}{l}\text { PAINCETON } \\ \text { PLABMA PHYSICS } \\ \text { LABOAATOAY }\end{array}\right.$

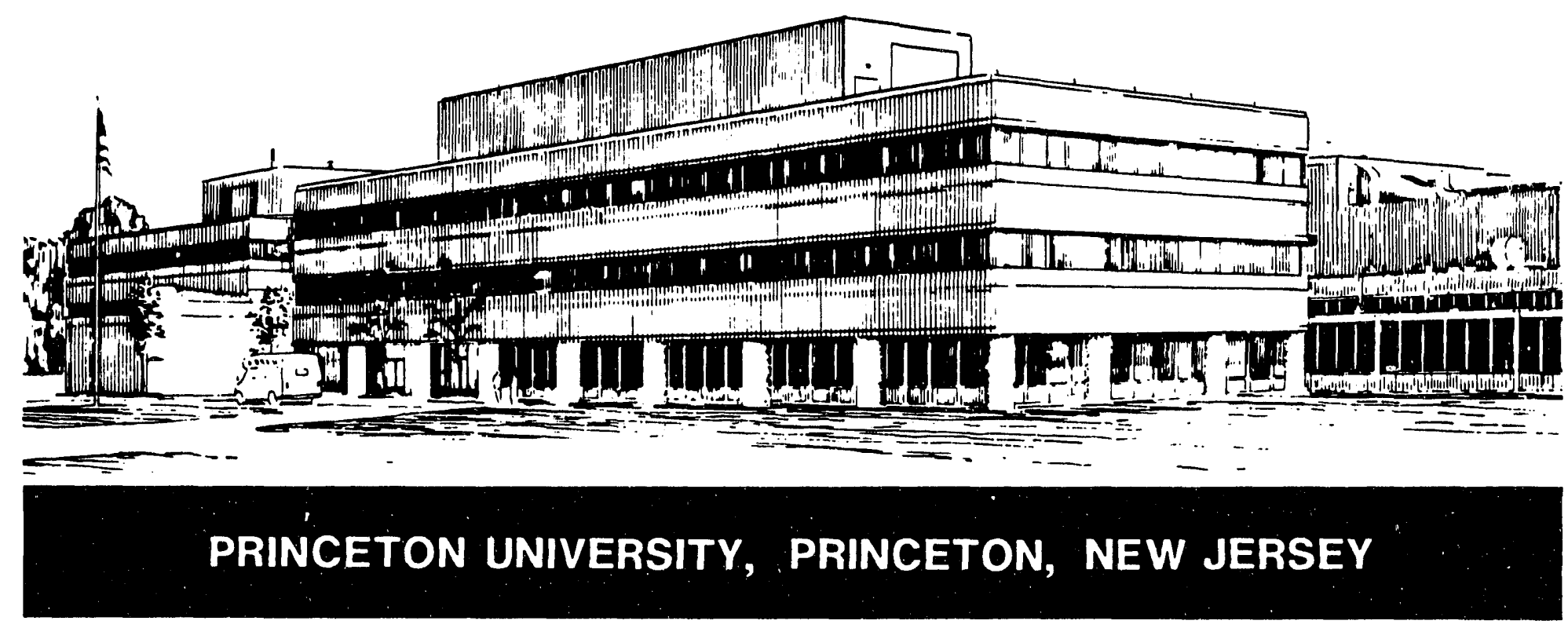




\section{NOTICE}

This report was prepared as an account of work sponsored by an agency of the United States Government. Neither the United States Government nor any agency thereof, nor any of their employees, makes any warranty, express or implied, or assumes any legal liability or responsibility for the accuracy, completeness, or usefulness of any information, apparatus, product, or process disclosed, or represents that its use would not infringe privately owned rights. Reference herein to any specific commercial produce, process, or service by trade name, trademark, manufacturer, or otherwise, does not necessarily constitute or imply its endorsement, recommendation, or favoring by the United States Government or any agency thereof. The views and opinions of authors expressed herein do not necessarily state or reflect those of the United States Government or any agency thereof.

\section{NOTICE}

This report has been reproduced from the best available copy. Available in paper copy and microfiche.

Number of pages in this report: 39

DOE and DOE contractors can obtain copies of this report from:

Office of Scientific and Technical Information

P.O. Box 62

Oak Ridge, TN 37831 ;

(615) $576-8401$.

This report is publicly available from the:

National Technical Information Service

Department of Commerce

5285 Port Royal Road

Springfield, Virginia 22161

(703) $487-4650$ 


\title{
Theory of Ballooning-Mirror Instabilities for Anisotropic Pressure Plasmas in the Magnetosphere
}

\author{
C. Z. Cheng and Q. Qian \\ Princeton Plasma Physics Laboratory \\ Princeton University, P. O. Box 451, Princeton, NJ 08543
}

\begin{abstract}
This paper deals with a kinetic-MHD eigenmode stability analysis of low frequency ballooning-mirror instabilities for anisotropic pressure plasmas in the magnetosphere. The ballooning mode is a dominant transverse wave driven unstable by pressure gradient in the bad curvature region (i.e., $(\vec{\kappa} \bullet \nabla \psi)(\nabla P \bullet \nabla \psi)>0$, where $\vec{\kappa}$ is the magnetic field curvature and $\psi$ is the magnetic flux function) with a dominant transverse magnetic field perturbation. The mirror mode with a dominant compressional magnetic field perturbation is excited when the product of plasma beta and pressure anisotropy (the ratio of the perpendicular pressure to the parallel pressure) is large. The field-aligned eigenmode equations take into account the coupling of the transverse and compressional components of the perturbed magnetic field and describe the coupled ballooningmirror mode. Because the energetic trapped ions precess very rapidly across the $\vec{B}$ field, their motion becomes very rigid with respect to low frequency MHD perturbations with symmetric structure of parallel perturbed magnetic field $\delta \mathrm{B}_{\|}$and electrostatic potential $\Phi$ along the north-south ambient magnetic field, and the symmetric ballooning-mirror mode is shown to be stable. On the other hand, the ballooning-mirror mode with antisymmetric $\delta \mathrm{B}_{\|}$and $\Phi$ structure along the northsouth ambient magnetic field is only weakly influenced by energetic trapped particle kinetic effects due to rapid trapped particle bounce motion and has the lowest instability threshold determined by MHD theory. With large plasma beta $\left(\beta_{\|} \geq O(1)\right)$ and pressure anisotropy $\left(P_{\perp} / P_{\|}>1\right)$ at equator the antisymmetric ballooning-mirror mode structures resemble the field-aligned wave structures of the multisatellite observations of a long lasting compressional Pc 5 wave event during November 14-15, 1979 [Takahashi et al., 1987a]. The study provides the theoretical basis for identifying the internal excitation mechanism of ULF (Pc 4-5) waves by comparing the plasma stability parameters computed from the satellite particle data with the theoretical values.
\end{abstract}

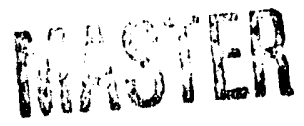




\section{Introduction}

Observation and theories of magnetic pulsations have been studied for more than three decades since they were first reported in the 1960's [Judge and Coleman, 1962]. The magnetic pulsations have been classified through their frequencies, waveforms, dominant magnetic components (transverse or compressional waves), and the associated plasma and geomagnetic conditions. These morphological features provide clues for understanding their excitation mechanisms (internally-driven instabilities or externally-driven Alfvén waves). To understand the physics of the magnetic pulsations, it is important to identify their excitation mechanisms. The external excitation mechanism involves local field-line resonance of standing shear Alfvén waves in response to the propagation of external disturbances. The externally excited PC 4-5 waves have azimuthal polarization with small azimuthal mode numbers on the order 10 or less and will not be discussed in the paper. Instead, we will concentrate on the theory of ultra-low frequency (ULF, waves driven internally by MHD type instabilities such as ballooning and mirror modes.

The internally driven ULF waves usually have large azimuthal mode numbers on the order of 100 and are mainly observed in the magnetosphere by satellites [Takahashi, 1988]. ULF compressional Pc 5 magnetic perturbations [Walker et al., 1982; Allan et al., 1982: Nagano and Araki, 1983; Misra et al., 1985; Lin and Barfield, 1985; Takahashi et al., 1985a, 1985b, 1987: Higuchi et al., 1986] with periods in the range of 150-600 seconds observed around the earth's magnetic equator near geosynchronous orbit were helieved to be driven by rnirror-type instabilities [Hasegawa, 1969; Cheng and Lin, 1987]. These highly compressional magnetic perturbations are usually observed during periods of enhanced ring current intensity and are associated with high beta, anisotropic pressure plasmas with $P_{\perp}>P_{\|}$, where the subscripts $\perp$ and $\|$ denote the components perpendicular and parallel to the ambient magnetic field respectively. The PC 5 walves have large azimuthal (east-west) wave numbers ( typically, m 50). Significant progress was. made in the multisatellite ( SCATHA, GOES2, GOES3, GEOS2) observations of a long lasting compressional Pc 5 wave event during November 14-15, 1979 |Takahashi el al., 1987: Takahashi, 1988] which revealed the field-aligned structures of the compressional magnetic field $\delta \mathrm{B}_{\|}$to be antisymmetric with respect to the earth's magnetic equator with a full latitudinal range ()! about $20^{\circ}$. The smaller transverse magnetic components, radial component $\delta \mathrm{B}_{\psi}$ and azimuthal component $\delta \mathrm{B}_{\phi}$, have a symmetric parity and their polarization varies with the magnetic latitude. The field-aligned structures of the three perturbed magnetic field components were shown in Fig. 14(b) in the paper by Takahashi et al. [1990]. The wave frequencies are about one order of magnitude smaller than the shear Alfvén frequency obtained from the measured plasma density and wave structures along the ambient magnetic field at geosynchronous orbit. The internally driven 
transverse waves usually have low frequency and dominant radial polarization, and they are believed to have similar (but more extended) field-aligned wave symmetry properties as the compressional Pc 5 waves [Engebretson et al., 1988].

Linear theories of internally driven hydromagnetic instabilities have been presented in terms of (1) mirror or drift mirror instabilities due to pressure anisotropy [Hasegawa, 1969; Pokhotelot et al., 1986; Cheng and Lin, 1987], (2) drift compressional instabilities due to pressurt nonuniformity [Hasegawa, 1971; Ng et al. 1984], and (3) trapped particle bounce and magnetic drift resonance excitation of ULF waves [Southwood, 1976; Chen and Hasegawa, 1991]. Two coupled eigenmode equations were derived [Cheng and Lin, 1987; Cheng, 1991] to describe the coupling between the transverse and the compressional magnetic field components. The transverse magnetic field equation shows that the shear Alfvén type wave can be destabilized by the combined effect of the magnetic field curvature and the plasma pressure gradient to excite the ballooning mode. The compressional magnetic field equation indicates that the mirror mode cill be destabilized by the combined effect of plasma beta and pressure anisotropy $\left(P_{-i}>P_{\|}\right)$. The coupling between the compressional and transverse magnetic field components is due to fintte perpendicular pressure gradient and the trapped particle perturbed pressures. By analyzing the mirror instability from the compressional wave equation both analytically and numericilly, Cheng and Lin [1987] was able to explain successfully the multisatellite observation of antisymmetric field-aligned structure of the compressional magnetic field of Pc 5 waves during a large substorm [Takahashi et al., 1987; Takahashi et al., 1990] in the magnetospheric ring current region where the plasma perpendicular pressure is larger than the parallel pressure. Recently, Chen and Hasegawa [1991] proposed that the perturbed compressional magnetic field with antisymmetric field-aligned structure can be excited by the drift Alfvén ballooning mirror instability thist is destabilized by hot particle pressure gradient via the magnetic drift-bounce resonance and may have lower pressure anisotropy threshold than the mirror instability.

However, comprehensive analytical and numerical eigenmode analyses that take into accoum the coupling between the transverse and the compressional magnetic field components in anisotropic pressure plasmas along the ambient magnetic field have still not yet been performed. The kinetic effects associated with the energetic trapped ions play an essential role in determining the stability of the ballooning-mirror mode. In this paper we present a kinetic-MHD eigenmode analysis of the ballooning-mirror mode along the ambient magnetic field. If the energetic tripped ion kinetic effects are neglected completely, the eigenmode equations are essentially the MHD eigenmode equations. Without the kinetic effects the MHD mode with symmetric $\delta B_{\|}$field. aligned structure has the lowest instability beta threshold. However, if the energetic trapped ion 
kinetic effects are included, the ballooning-mirror mode with symmetric $\delta \mathrm{B}_{\|}$and $\Phi$ field-aligned structure is completely stabilized. The stabilization results from the fact that the hot trapped jons precess very rapidly across the $\vec{B}$ field, their motion becomes very rigid with respect to lou frequency $M H D$ perturbations with symmetric structure of parallel perturbed magnetic field $\delta B_{\|}$ and electrostatic potential $\Phi$ along the north-south ambient magnetic field, and thus they do not contribute to the destabilizing pressure gradient to drive ballooning instability. Or the other hand. the energetic trapped ion's rapid bounce motion tends to average out the kinetic contribution associated with the antisymmetric $\delta B_{\|}$and $\Phi$ wave structure along the north-south ambiem magnetic field. Therefore, the antisymmetric ballooning-mirror mode is only weakly influenced by energetic trapped particle kinetic effects and has the lowest instability beta threshold mainly determined by MHD theory. The results not only explain the observed wave structures in terms of predicted plasma properties, but also provide new ways of identifying the internal excitition mechanism of observed ULF waves.

The paper is organized as follows. Section 2 reviews the kinetic-MHD model for describing the stability of low frequency MHD type modes. The kinetic-MHD equilibrium, the kinetic-MHD eigenmode equations for the ballooning-mirror mode, and a quadratic form thall can provide significant insight to the stability properties are presented. The theoretical investigation of the ballooning-mirror instabilities in a dipole magnetic field is given in Sec. 3. In particular, a locial MHD stability theory, a field-aligned MHD eigenmode analysis, and the energetic trapped particle stabilization of symmetric $\delta \mathrm{B}_{\|}$modes are presented. Finally, a summary and discussion is given in Sec. 4.

\section{Kinetic-MHD Model for Low Frequency MHD Modes}

\subsection{Kinetic-MHD Equilibrium}

If the plasma convection in the magnetosphere is small, the magnetospheric equilibrium ciul be approximated by a static MHD equilibrium with anisotropic pressure, which is described by the system of equations

$$
\begin{aligned}
& \overrightarrow{\mathbf{J}} \times \overrightarrow{\mathrm{B}}=\nabla \cdot \overrightarrow{\mathrm{P}}=\nabla \mathrm{P}_{\perp}-\nabla \bullet\left[\left(\mathrm{P}_{\perp}-\mathrm{P}_{\|}\right) \hat{\mathrm{b}} \hat{\mathrm{b}}\right] \\
& \nabla \times \overrightarrow{\mathrm{B}}=\overrightarrow{\mathbf{J}} \\
& \nabla \bullet \overrightarrow{\mathrm{B}}=0
\end{aligned}
$$


where $\hat{b}$ is a unit vector along an equilibrium magnetic field line, and $\vec{J}, \vec{B}$, and $\vec{P}$ are the equilibrium current, magnetic field, and pressure tensor, respectively.

Considering a general three dimensional magnetospheric equilibrium with nested flux surfaces, the magnetic field can be expressed as $\vec{B}=\nabla \psi \times \nabla \alpha$, where $\psi$ is chosen as the magnetic flux function. Both $\psi$ and $\alpha$ are constant along magnetic field lines. The lines where surfaces of constant $\psi$ and $\alpha$ intersect represent magnetic field lines. Note that $\psi$ must be a periodic function of toroidal angle $\phi$ in cylindrical $(R, \phi, Z)$ coordinate to ensure periodicity constraint. In terms of a flux coordinate system $(\psi, \Theta, \phi)$ with $\Theta$ being the generalized poloidal angle varying between 0 and $2 \pi, \alpha$ can be expressed as $\alpha=\phi-q(\psi) \Theta-\Delta\left(\psi_{s} \Theta, \phi\right)$ without loss of generality, where $\Delta(\psi, \Theta, \phi)$ is periodic in both $\Theta$ and $\phi$. For a collisionless plasma the particle energy $\left(\varepsilon=v^{2} / 2\right)$ and the adiabatic invariants, magnetic moment $\left(\mu=v_{\perp}{ }^{2} / 2 B\right)$ and the longitudinal invariant $\left(J_{\|}=\int d s\right.$ $v_{\|}$), are constant during the drift motions, where $v_{\|}$and $v_{\perp}$ are the components of the velocity parallel and perpendicular to $\overrightarrow{\mathrm{B}}$, respectively. The guiding center equilibrium particle distribution function must have the form $F=F\left(\varepsilon, \mu, J_{\|}\right)$. In general, $J_{\|}=J_{\|}(\varepsilon, \mu, \psi, \alpha)$ and $F=F(\varepsilon, \mu, \psi, \alpha)$. If all particles on each field line share the same drift surface, where $\psi$ labels the drift surface, then $\mathrm{J}_{\|}=\mathrm{J}_{\|}(\varepsilon, \mu, \psi)$ and $\mathrm{F}=\mathrm{F}(\varepsilon, \mu, \psi)$. The guiding-center particle distributions $\mathrm{F}(\varepsilon, \mu, \psi)$ can be eithel prescribed by an analytical form or obtained from the satellite measurements of the particle flux.

The equilibrium parallel and perpendicular pressures are given by

$$
\left(\begin{array}{l}
P_{\|} \\
P_{\perp}
\end{array}\right)=\sum_{j, \sigma_{\|}} 2 \pi M_{j} \int_{0}^{\infty} \mathrm{d} \varepsilon \int_{0}^{\varepsilon / B} \mathrm{~d} \mu\left[B F_{j} /\left|v_{\|}\right|\right]\left(\begin{array}{c}
2(\varepsilon-\mu B) \\
\mu B
\end{array}\right)
$$

where the summation is over the particle species $\mathrm{j}$ and $\sigma_{\|}$which represents the direction of particle velocity parallel to $\vec{B}$, and $M_{j}$ is the particle mass. The parallel velocity $v_{\|}$has the form

$$
v_{\|}=\sigma_{\|} \sqrt{2}(\overline{\varepsilon-\mu B})
$$

By inspection $\mathrm{P}_{\perp}$ and $\mathrm{P}_{\|}$are functions of $\psi$ and $\mathrm{B}$ only.

It is convenient to introduce the functions 


$$
\sigma \equiv 1-(1 / \mathrm{B})\left(\partial \mathrm{P}_{\|} / \partial \mathrm{B}\right)_{\psi},
$$

and

$$
\tau \equiv 1+(1 / \mathrm{B})\left(\partial \mathrm{P}_{\perp} / \partial \mathrm{B}\right)_{\psi}
$$

If $\sigma>0$ and $\tau>0$ are satisfied everywhere in the plasma, the magnetosphere is stable to the wellknown MHD "firehose" and "mirror" instabilities, respectively [Grad, 1967]. The momentum balance equation parallel to the equilibrium magnetic field is given by

$$
\vec{B} \cdot \nabla P_{\|}=\left(P_{\|}-P_{\perp}\right) \hat{b} \cdot \nabla B \text {. }
$$

Making use of Eqs. (6)-(8), the momentum balance equation perpendicular to the magnetic field is given by

$$
\tau \nabla_{\perp}\left(\mathrm{B}^{2} / 2\right)+\tilde{\nabla}_{\perp} \mathrm{P}_{\perp}=\overrightarrow{\mathrm{K}} \sigma \mathrm{B}^{2}
$$

where $\widetilde{\nabla}=\nabla-\nabla \mathrm{B}(\partial / \partial \mathrm{B})_{\psi}, \overrightarrow{\mathrm{K}}=\hat{\mathrm{b}} \bullet \nabla \hat{\mathrm{b}}$ is the magnetic field curvature. Note that the parallel momentum balance equation, Eq. (8), is automatically satisfied if the particle distribution $F(\varepsilon, \mu, \psi)$ is used to compute $P_{\perp}$ and $P_{\|}$. From Eq. (8), $\sigma$ can be simplified as $\sigma=1+\left(P_{\perp}-P_{\|}\right) / B^{2}$.

\subsection{Kinetic-MHD Eigenmode Equations}

The linearized kinetic-MHD eigenmode equations for the low-frequency transverse and compressional Alfvén waves had been derived in the limit of zero parallel electric field / Cheng and Lin, 1987; Cheng, 1991]. The perturbed transverse magnetic field is related to the electrostatic potential $\Phi$ by $\delta \overrightarrow{\mathrm{B}}_{\perp} \approx \nabla \times\left[\overrightarrow{\mathrm{B}}\left(\overrightarrow{\mathrm{B}} \odot \nabla \Phi / \omega \mathrm{B}^{2}\right)\right]$, where $\omega$ is the eigenmode frequency of the perturbed quantities which have a temporal dependence of $\exp (-\mathrm{i} \omega \mathrm{t})$. By assuming that the perpendiculit wavelength is shorter than the parallel wavelength which is in turn shorter than the equilibrium scale length $\left(k_{\perp} \gg k_{\|} \gg 1 / L\right)$ and the particle gyroradius is small compared to the perpendiculal wavelength $\left(k_{\perp} \rho_{L} \ll 1\right)$ for all particle species, the perturbed guiding center particle distribution $\delta i$ is determined from the drift kinetic equation. If the perturbed particle guiding center distribution is written in terms of a nonadiabatic particle distribution function $\mathrm{g}$ by

$$
\delta f=-\frac{i c}{\omega B^{2}} \vec{B} \times \nabla \Phi \cdot \nabla F-\frac{\mu}{B^{2}} \frac{\partial F}{\partial \mu} \vec{B} \cdot \delta \vec{B}+\hat{g}
$$


the perturbed pressures can be written as

$$
\left(\begin{array}{c}
\delta \mathrm{p}_{\|} \\
\delta \delta \mathrm{p}_{\perp}
\end{array}\right)=-\frac{\mathrm{i} \overrightarrow{\mathrm{B}} \times \nabla \Phi}{\omega \mathrm{B}^{2}} \cdot \tilde{\nabla}\left(\begin{array}{c}
\mathrm{P}_{\|} \\
\mathrm{P}_{\perp}
\end{array}\right)+\frac{\overrightarrow{\mathrm{B}} \cdot \delta \overrightarrow{\mathrm{B}}}{\mathrm{B}}\left(\frac{\partial}{\partial \mathrm{B}}\right)_{\psi}\left(\begin{array}{c}
\mathrm{P}_{\|} \\
\mathrm{P}_{\perp}
\end{array}\right)+\left(\begin{array}{c}
\delta \hat{\mathrm{p}}_{\|} \\
\delta \hat{\mathrm{p}}_{\perp}
\end{array}\right)
$$

where $\tilde{\nabla}=\nabla-\nabla \mathrm{B}(\partial / \partial \mathrm{B})_{\psi}$, and the kinetic effect due to trapped particles is included in the nonadiabatic perturbed pressures $\delta \hat{\mathrm{p}}_{\perp}$ and $\delta \hat{\mathrm{p}}_{\|}$which are given by

$$
\left(\begin{array}{c}
\delta \hat{\mathrm{p}}_{\|} \\
\delta \hat{\mathrm{p}}_{\perp}
\end{array}\right)=\sum_{j} \mathrm{M} \int \mathrm{d}^{3} v \hat{\mathrm{g}}\left(\begin{array}{c}
2(\varepsilon-\mu B) \\
\mu B
\end{array}\right)
$$

In the right-hand side of Eq. (11), the first term represents the convective derivative of plasmi pressure, and the second term represents the compressional field effect associated with pressure nonuniformity along the field line. In the absence of parallel electric field, the drift kinetic equation for the linearized nonadiabatic particle distribution function $\hat{g}$ is given by

$$
\left[\frac{\partial}{\partial t}+\left(\vec{v}_{\|}+\vec{v}_{d}\right) \cdot \nabla\right] \hat{g}=\left[\frac{e}{M} \frac{\partial F}{\partial \varepsilon} \frac{\partial}{\partial t}-\frac{c \vec{B} \times \nabla F}{B^{2}} \cdot \nabla\right] Y,
$$

where $\mathrm{Y}=\mathrm{i} \overrightarrow{\mathrm{v}}_{\mathrm{d}} \bullet \nabla \Phi / \omega-\mathrm{M} \mu \delta \mathrm{B}_{\|} / \mathrm{e}, \overrightarrow{\mathrm{v}}_{\mathrm{d}}=\left(\mathrm{McB} / \mathrm{eB}^{2}\right) \times\left[\nabla(\mu \mathrm{B})+\vec{\kappa} \mathrm{v}_{\|}{ }^{2}\right]$ is the particle magnetic drift velocity in the equilibrium magnetic field, and $\vec{K}=(\vec{B} / B) \bullet \nabla(\vec{B} / B)$ is the equilibrium magnetic field curvature. In terms of a Fourier series representation the linearized nonadiabatic particle distribution function $\hat{\mathrm{g}}$ has been derived for a magnetospheric geometry in the paper b: Cheng [1991] (see Sec. 4 and Appendix C) and will not be repeated here.

We will consider a WKB wave form perpendicular to the ambient magnetic field so that $\nabla$. operates only on the perturbed quantities and $\nabla_{\perp}=i \vec{k}_{\perp}$. The eigenmode equations for $\hat{\Phi}(=\Phi / \omega$ ) and $\delta B_{\|}$can obtained from Eqs. (40), (41), and (45) in the paper by Cheng $|1991|$ and are given by

$$
\overrightarrow{\mathrm{B}} \cdot \nabla\left(\frac{\sigma \mathrm{k}_{\perp}^{2}}{\mathrm{~B}^{2}} \overrightarrow{\mathrm{B}} \cdot \nabla \hat{\Phi}\right)+\frac{\rho \mathrm{k}_{\perp}^{2} \omega^{2}}{\mathrm{~B}^{2}} \hat{\Phi}
$$




$$
+\frac{(\vec{B} \times \vec{k})}{B^{2}} \bullet \vec{k}_{\perp}\left[\frac{\vec{B} \times \tilde{\nabla} P_{\|}}{B^{2}} \bullet \vec{k}_{\perp} \hat{\Phi}+\sigma B \delta B_{\|}-\delta \hat{p}_{\|}\right]=0
$$

and

$$
\begin{aligned}
\overrightarrow{\mathrm{B}} \bullet \nabla\left[\frac{\sigma}{\mathrm{B}^{2}} \overrightarrow{\mathrm{B}} \bullet \nabla \delta \mathrm{B}_{\|}\right] & +\frac{\rho \omega^{2}}{\mathrm{~B}^{2}} \delta \mathrm{B}_{\|} \\
& -\mathrm{k}_{\perp}^{2}\left[\tau \delta \mathrm{B}_{\|}-\overrightarrow{\mathrm{k}}_{\perp} \cdot \frac{\overrightarrow{\mathrm{B}} \times \tilde{\nabla} \mathrm{P}_{\perp}}{\mathrm{B}^{3}} \hat{\Phi}+\frac{\delta \hat{\mathrm{p}}_{\perp}}{\mathrm{B}}\right]=0,
\end{aligned}
$$

where $\rho$ is the mass density, and $\tilde{\nabla}=\nabla-\nabla B(\partial / \partial \mathrm{B})_{\psi}$.

Equations (14) and (15) describe the coupling between the compressional and transverse perturbed magnetic field due to finite magnetic field curvature, prerpendicular pressure gradient, and the nonadiabatic trapped particle kinetic pressure responses $\delta \hat{\mathrm{p}}_{\perp}$ and $\delta \hat{\mathrm{p}}_{\|}$. From Eq. (14) we see that the transverse shear Alfvén wave in a high $\beta(=O(1))$ plasma can be destabilized by the pressure gradient in a bad magnetic field curvature with $(\vec{K} \bullet \nabla \psi)(\nabla P \bullet \nabla \psi)>0$ to excite ballooning modes. Equation (15) indicates that the compressional Alfvern waves can be destabilized by the pressure anisotropy $(\tau<0)$ to excite mirror modes. The eigenmode structure of the mirror instability was first obtained by analyzing Eqs. (14) and (15) perturbatively. The $\delta \mathrm{B}_{\|}$is obtained from Eq. (15) by neglecting electrostatic potential but including trapped particle effects /Cheng and Lin, 1987; Takahashi et al., 1990]. The transverse magnetic field is then obtained from Ec]. (14) by neglecting the magnetic field curvature term. In general, to obtain quantitative eigensolutions of the coupled eigenmode equations, extensive numerical works are required.

\subsection{Quadratic Form}

A quadratic form can be derived from Eqs. (14) and (15) and provides significant insights to the stability properties. Assuming that the perturbed quantities vanish at the end points of the field line, we obtain the quadratic form

$$
D(\omega)=\delta K-\delta W_{f}-\delta W_{k}=0
$$

where the inertia energy is given by 


$$
\delta \mathrm{K}=\omega^{2} \int \frac{\mathrm{ds}}{\mathrm{B}} \frac{\rho}{\mathrm{B}^{2}}\left[\mathrm{k}_{\perp}^{2}|\hat{\Phi}|^{2}+\frac{\left|\delta \mathrm{B}_{\mid}\right|^{2}}{\mathrm{k}_{\perp}^{2}}\right]
$$

and $s$ is the distance along the field line. The fluid potential energy is given by

$$
\begin{aligned}
\delta \mathrm{W}_{\mathrm{f}}=\int \frac{\mathrm{ds}}{\mathrm{B}}\left\{\frac{\sigma}{\mathrm{B}^{2}} \mathrm{k}_{\perp}^{2}|\overrightarrow{\mathrm{B}} \cdot \nabla \hat{\Phi}|^{2}+\frac{\sigma}{\mathrm{B}^{2} \mathrm{k}_{\perp}^{2}}\left|\overrightarrow{\mathrm{B}} \cdot \nabla \delta \mathrm{B}_{\|}\right|^{2}\right. \\
\left.+\tau\left|\delta \mathrm{B}_{\|}-\frac{\overrightarrow{\mathrm{k}}_{\perp} \times \overrightarrow{\mathrm{B}}}{\tau \mathrm{B}^{3}} \cdot \tilde{\nabla} \mathrm{P}_{\perp} \hat{\Phi}\right|^{2}-\alpha_{\mathrm{p}}\left|\frac{\mathrm{k}_{\perp} \hat{\Phi}}{\mathrm{L}}\right|^{2}\right\}
\end{aligned}
$$

with

$$
\alpha_{p}=\left(\frac{\vec{k}_{\perp} \times \vec{B} \bullet \vec{k}}{k_{\perp} B}\right) \frac{\vec{k}_{\perp} \times \vec{B}}{k_{\perp} B^{3}} \cdot\left[\left(\frac{\sigma}{\tau}\right) \tilde{\nabla} P_{\perp}+\widetilde{\nabla} P_{\|}\right] L^{2}
$$

The kinetic potential energy due to all species is

$$
\delta \mathrm{W}_{\mathrm{k}}=\int \frac{\mathrm{ds}}{\mathrm{B}}\left\{\left[\delta \mathrm{B}_{\|}+\frac{\overrightarrow{\mathrm{k}}_{\perp} \bullet \overrightarrow{\mathrm{B}} \times \nabla\left(\mathrm{B}^{2} / 2\right)}{\mathrm{B}^{3}} \hat{\Phi}\right]^{*} \frac{\delta \hat{\mathrm{p}}_{\perp}}{\mathrm{B}}+\frac{\overrightarrow{\mathrm{k}}_{\perp} \cdot \overrightarrow{\mathrm{B}} \times \mathrm{k}}{\mathrm{B}^{2}} \hat{\Phi}^{*} \delta \hat{\mathrm{p}}_{\|}\right\}
$$

In deriving the quadratic form we have made use of the equilibrium relation, Eq. (9). In obtaining Eq. (18) a term in the order of $\left(\rho \omega^{2} / \mathrm{B}^{2}-\mathrm{k}_{\|}{ }^{2}\right) / \mathrm{k}_{\perp}{ }^{2}$ is ignored. An approximation, $\delta \mathrm{B}_{\|}-$ $\overrightarrow{\mathrm{k}} \perp \times \overrightarrow{\mathrm{B}} \cdot \tilde{\nabla} \mathrm{P}_{\perp} \hat{\Phi} / \tau \mathrm{B}^{3} \approx-\delta \hat{\mathrm{p}}_{\perp} / \mathrm{B}$, is also made in obtaining the second $\delta \hat{\mathrm{p}}_{\perp}$ term in Eq. (2()). Equation (18) clearly displays the various fluid free energy sources. In particular, if $\sigma>0)\left(i . e ., 1+\left(P_{\perp}-\right.\right.$ $\left.\mathrm{P}_{\|}\right) / \mathrm{B}^{2}>0$ ) and $\tau>0$ (i.e., $1+(1 / \mathrm{B})\left(\partial \mathrm{P}_{\perp} / \partial \mathrm{B}\right)_{\psi}>0$ ) are satisfied everywhere in the plasma, the magnetosphere is stable to the well-known MHD "firehose" and "mirror" instabilities, respectively [Grad, 1967]. The third term in Eq. (18) drives ballooning modes by pressure gradient in the bad curvature region where $\alpha_{p}>0$.

The quadratic form, Eq. (16), is not Hermitian, and there is no energy principle. However, if $\delta W_{k}$ is small compared with $\delta W_{f}$ and can be neglected, then Eq. (16) is an energy principle and the variational technique can be apolied to obtain eigenvalue and eigenfunction. In the magnetospheric ring current region, it had been shown by Cheng and Lin $|1987|$ that $\delta W_{k}$ is small compared with $\delta \mathrm{W}_{\mathrm{f}}$ for perturbations with $\widehat{\Phi}$ and $\delta \mathrm{B}_{\|}$having antisymmetric structures along 
the north-south symmetric equilibrium magnetic field lines in the low frequency limit with $\left(\omega-\omega_{d}\right.$ ) $<<\omega_{b}$, where $\omega_{d}=<\vec{k}_{1} \cdot \vec{v}_{d}>$ is the trapped particle bounce-averaged magnetic drift frequency and $\omega_{b}$ is the bounce frequency. In this limit $\delta W_{k}$ is of the order of $\left(\omega / \omega_{b}\right)$ smaller than $\delta W_{l}$ since the contributions from the $\left(\omega-\omega_{d}\right)=0$ magnetic drift-bounce resonance vanishes. Therefore, if $\delta W_{k}$ can be neglected, two parameters, $\alpha_{p}$ and $\tau$, will determine the MHD stability. In the limit that the perturbed transverse and the perturbed compressional magnetic field components decouple. it is possible that the ballooning instability can occur with perturbation localized around the region where $\alpha_{\mathrm{p}}>0$, and the mirror mode can be unstable with perturbation localized around the region where $\tau<0$. In general, the transverse and the compressional perturbed magnetic field components couple, and the stability properties wil be modified.

In the limit $\left(\omega-\omega_{d}\right) \approx \omega_{b}$ the drift-bounce resonance can contribute to $\delta W_{k}$, and Chen and Hasegawa [1991] proposed that the drift Alfvén ballooning-mirror instability can be destabilized by hot particle pressure gradient via the magnetic drift-bounce resonance in the form of compressional magnetic field with antisymmetric field-aligned structure. The drift Alfvén ballooning mirror instability may have lower pressure anisotropy threshold than the mirror instability. However. wave resonance with all particle species must be included in the calculation before a definite answer on the stability threshold can be concluded.

\section{Theory of Ballooning-Mirror Instabilities in a Dipole Magnetosphere}

In this section we study the stability of ballooning and mirror modes in the magnetosphere. Let us consider a magnetospheric plasma consisting of a core (c) background component and a hot (h) bi-Maxwellian ion component with the hot plasma density smaller than the core plasma density. $n_{h} \ll n_{c}$, and the hot plasma temperature larger than the core plasma temperature, $T_{h}>T_{c}$. The plasma beta is dominated by the hot component. For the ring current plasma near the geosynchronous orbit, typically the core plasma temperature is $T_{c} \approx 10 \mathrm{eV}$, the hot plasmil temperature is $T_{h} \approx 10^{4} \mathrm{eV}$, the ratio of the hot plasma density to the core plasma density is ( $n_{h /} / n_{c}$ ) $\approx 10^{-1}$, thus the ratio of core plasma beta to the hot plasma beta is $\left(\beta_{c} / \beta_{h}\right)=10^{-2}$. The equatorial magnetic field is approximately $100 \mathrm{nT}$. The hot proton Larmor radius $\rho_{\mathrm{L}}$ is approximately 1()() $\mathrm{km}$, and for an azimuthal wave number $\mathrm{m} \approx 10^{2}$ we have $\left(\mathrm{k}_{\perp} \rho_{\mathrm{L}}\right)^{2} \approx\left(2.5 \times 10^{-3} \mathrm{~m}\right)^{2}=0.062 .5 \ll$ 1. Therefore, the hot ion FLR effect can be neglected. 
The bi-Maxwellian guiding center velocity distribution for energetic ion is chosen it the magnetic equator, expressed in terms of adiabatic invariant and constants of motion variables, and is given by

$$
\begin{aligned}
F(\varepsilon, \mu, \psi) & =N(\psi)\left[2 \pi T_{\|}(\psi) / M\right]^{-3 / 2} \exp \left[-M v_{\|}^{2} / T_{\|}(\psi)-M v_{\perp}^{2} / T_{\perp}(\psi, B)\right] \\
& =N(\psi)\left[2 \pi T_{\|}(\psi) / M\right]^{-3 / 2} \exp \left[-M \varepsilon / T_{\|}(\psi)+M \mu B_{0}(\psi) / T_{0}(\psi)\right],
\end{aligned}
$$

where $M$ is the particle mass, and the temperature ratio is $T_{\perp}\left(\psi, B / T_{\|}(\psi)=\left|1-B_{0} T_{\|} / B T_{0}\right|^{-1}\right.$. $B_{0}(\Psi)$ can be chosen as the magnetic field at the magnetic equator. The particle density is given by $\mathrm{n}(\psi, \mathrm{B})=\mathrm{N}(\psi)\left[\mathrm{T}_{\perp}(\psi, \mathrm{B}) / \mathrm{T}_{\|}(\psi)\right]$, the parallel pressure is given by $\mathrm{P}_{\|}(\psi, \mathrm{B})=\mathrm{P}(\psi) \mathrm{T}_{\perp}(\psi, \mathrm{B}) / \mathrm{T}_{\|}(\psi)$, thie perpendicular pressure is given by $P_{\perp}(\psi, B)=P(\psi)\left[T_{\perp}(\psi, B) / T_{\|}(\psi)\right]^{2}$. From Eq. (7) the pressure anisotropy parameter is given by $\tau=1+\left(2 \mathrm{P}_{\perp} / \mathrm{B}^{2}\right)\left[1-\mathrm{P}_{\perp} / \mathrm{P}_{\|}\right]$, where $\mathrm{N}(\psi)$ and $\mathrm{P}(\psi)$ $=\mathrm{N}(\psi) \mathrm{T}_{\|}(\psi)$ are the density and pressure in the isotropic pressure limit, respectively. From $\mathrm{E}(\mathrm{f}$. (19) the ballooning instability parameter is given by $\alpha_{p}=\left(k_{\phi}{ }^{2} / k_{\perp}{ }^{2}\right)\left(\kappa_{c} L\right) \mid(\sigma / \tau)\left(P_{\perp} / B^{2}\right)$ $\left.\partial \ln \mathrm{P}_{\perp} / \partial \ln L+\left(\mathrm{P}_{\|} / \mathrm{B}^{2}\right) \partial \ln \mathrm{P}_{\mid} / \partial \ln L\right]$, where $\kappa_{c}=\vec{\kappa} \bullet \nabla \psi /|\nabla \psi|$ is the normal magnetic field curvature. Note that $\alpha_{\mathrm{p}}$ and $\tau$ are not linearly independent. Also note that for a bi-Maxwellian distribution $\partial \ln P_{\|} / \partial \ln L=\partial \ln P / \partial \ln L+\partial \ln \left(T_{\perp} / T_{\|}\right) / \partial \ln L$. If $T_{\|} / T_{0}$ is chosen to be a constant and $B_{\mathcal{O}}(L)$ is chosen to be the equatorial magnetic field, then $\partial \ln \left(\mathrm{T}_{\perp} / \mathrm{T}_{\|}\right) / \partial \ln \mathrm{L}=-3\left(\mathrm{~T}_{\perp} / \mathrm{T}_{\|}-1\right)$. Similarly, $\partial \ln \mathrm{P}_{\perp} / \partial \ln \mathrm{L}=$ $\partial \ln \mathrm{P} / \partial \ln \mathrm{L}+2 \partial \ln \left(\mathrm{T}_{\perp} / \mathrm{T}_{\|}\right) / \partial \ln \mathrm{L}$.

We will consider a dipole magnetic field $\vec{B}=\nabla \psi \times \nabla \phi$, where $\psi=-M_{E} \sin ^{2} \theta / r$, with $\mathrm{r}$ and $\theta$ being the radius and polar angle in the right-handed spherical coordinate system $(r, \theta, \phi)$, and $\mathrm{M}_{\mathrm{E}}$ is the dipole moment. The dipole magnetic field line equation is given by $r=\mathrm{L} \sin ^{2} \theta$, where $\mathrm{L}$ is the $\mathrm{L}$-shell distance in the equatorial plane. Note that $\psi=-\mathrm{M}_{\mathrm{E}} / \mathrm{L}$. The components of the dipole magnetic field are given by $B_{r}=-2 M_{E} \cos \theta / r^{3}, B_{\theta}=-M_{E} \sin \theta / r^{3}$, and the magnitude of the magnetic field is given by $B=B_{0}(\theta=0, L)\left(1+3 \cos ^{2} \theta\right)^{1 / 2} / \sin ^{6} \theta$ along the field line, where $B_{0}=B(\theta=0, r=L)$. The normal component of the magnetic field curvature is given by $\kappa_{c}=-3\left(1+\cos ^{2} \theta\right) /\left[\mathrm{L} \sin \theta\left(1+3 \cos ^{2} \theta\right)^{3 / 2}\right]$.

In the following we will assume that $\left(\omega-\omega_{d}\right)<<\omega_{b}$ and neglect small bounce resonance terms in $\delta \hat{\mathrm{p}}_{\perp}$ and $\delta \hat{\mathrm{p}}_{\|}$that are in the order of $\left(\omega / \omega_{\mathrm{b}}\right)$. In Sec. 3.1 we will first totally neglect $\delta \hat{\mathrm{p}}$. and $\delta \hat{\mathrm{p}}_{\|}$and discuss the local stability properties by considering the coupling between the ballooning-mirror modes. The corresponding eigenmode stability theory of the ballooning and the mirror modes that takes into account the field-aligned plasma properties is presented in Sec. 3.2. By totally neglecting $\delta \hat{\mathrm{p}}_{\perp}$ and $\delta \hat{\mathrm{p}}_{\|}$, the eigenmode equations are essentially MHD equations with 
anisotropic pressure along the field line. It is shown that the MHD ballooning modes with $\widehat{\Phi}$ and $\delta \mathrm{B}_{\|}$having symmetric structures along the north-south equilibrium magnetic field lines have the lowest beta stability threshold. The antisymmetric MHD modes have higher beta stability threshold. For small pressure anisotropy the antisymmetric MHD mode is a pure ballooning mode. But, for higher pressure anisotropy with simple mirror threshold $(\tau=0)$ exceeded all equator, the antisymmetric MHD mode becomes a hybrid ballooning-mirror mode with strong coupling between the transverse and the parallel magnetic field components. When the leading kinetic contribution from $\delta \hat{\mathrm{p}}_{\perp}$ and $\delta \hat{\mathrm{p}}_{\|}$is included in the analysis for the symmetric modes, the symmetric $\delta \mathrm{B}_{\|}$modes are shown numerically to be stabilized by the energetic trapped particle kinetic effect in the absence of wave-particle resonance. An analytical theory for the energetic trapped particle stabilization of the symmetric ballooning mode is given in Sec. 3.3. On the othes hand, the kinetic trapped particle effects on the stability of the antisymmetric $\delta B_{\|}$mode alle relatively small. Therefore, the antisymmetric MHD modes have the lowest critical beta threshold for instability and should account for the ULF wave events.

It is important to comment here that in the MHD theory unstable MHD instabilities have zero real frequency. The real frequency of the MHD instabilities must be determined by considering the Doppler shift frequency associated with the balk plasma convection as well as the diamagnetic and magnetic drift frequencies of various plasma species. As long as the frequency of the mode frequency, the core plasma diamagnetic drift frequencies, and the Doppler shift freyuency associated with the balk plasma convection are small compared with the hot ion magnetic drift and diamagnetic drift frequencies, the analysis performed in the paper is valid in the lowest order in the frequency ordering.

\subsection{Local Stability Theory}

A great deal of physical insight can be achieved by studying the local dispersion relation of the coupled eigenmode equations, Eqs. (14) and (15), near equator by neglecting the noniadiabiltic pressure responses $\delta \hat{\mathrm{p}}_{\perp}$ and $\delta \hat{\mathrm{p}}_{\|}$. In an axisymmetric magnetospheric geometry without toroidal magnetic field the perpendicular wave vector can be expressed as $\overrightarrow{\mathrm{k}}_{\perp}=\mathrm{k}_{\phi} \nabla \phi /|\nabla \phi|+\mathrm{k}_{\psi} \nabla \psi /|\nabla \psi|$. where $k_{\phi}=m / R$ is the azimuthal wave number, $m$ is the azimuthal mode number, and $k_{\psi}$ is the radial (across the L-shells) wave number. We will consider that the azimuthal wavelength is much shorter than the radial wavelength, i.e., $k_{\phi}>>k_{\psi}$. By assuming a WKB wave form along the field line so that $\hat{\Phi}$ and $\delta B_{\|}$ase proportional to $\exp \left(i k_{\|} s\right)$ where $s$ is the distance along the field line. Eqs. (14) and (15) reduce to 


$$
\begin{aligned}
& {\left[\omega^{2} / \mathrm{V}_{\mathrm{A}}^{2}-\sigma \mathrm{k}_{\|}{ }^{2}+\mathrm{K}_{\mathrm{c}} \mathrm{P}_{\|}{ }^{\prime} \sin ^{3} \theta / \mathrm{BB}_{0}\right] \hat{\Phi}+\left(\sigma \mathrm{k}_{\mathrm{c}} / \mathrm{k}_{\phi}\right) \delta \mathrm{B}_{\|}=0} \\
& {\left[\omega^{2} / \mathrm{V}_{\mathrm{A}}{ }^{2}-\sigma \mathrm{k}_{\|}{ }^{2}-\tau \mathrm{k}_{\phi}{ }^{2}\right] \delta \mathrm{B}_{\|}+\left(\mathrm{k}_{\phi}{ }^{3} \mathrm{P}_{\perp}{ }^{\prime} \sin ^{3} \theta / \mathrm{BB}_{0}\right) \hat{\Phi}=0}
\end{aligned}
$$

where $\kappa_{c}=\vec{K} \cdot \nabla \psi /|\nabla \psi|, P_{\|, \perp}{ }^{\prime}=\partial P_{\|, \perp} / \partial L$, and $V_{A}=B / \sqrt{\rho}$ is the Alfvén velocity, and the background plasma mass density $\rho$ is uniform along the field line. In the decoupling limit, $E$ l. (22) describes a purely growing ballooning mode with a dominant $\delta \vec{B}_{\perp}$ perturbation when $\omega_{K}{ }^{2} \equiv$ $\left(\sigma \mathrm{k}_{\|}{ }^{2}-\kappa_{\mathrm{c}} \mathrm{P}_{\|} \sin ^{3} \theta / \mathrm{BB}_{\mathrm{o}}\right) \mathrm{V}_{\mathrm{A}}{ }^{2}$ is negative, where the $\sigma \mathrm{k}_{\|}{ }^{2}$ term represents stabilizing field line bending effect for $\sigma>0$ and the $\kappa_{c} P_{\|}{ }^{\prime}$ term represents the destabilizing effect of pressure gradient in a 'bad' magnetic field curvature if $\kappa_{c} \mathrm{P}_{\|}$' $>0$. Equation (23) describes a purely growing mirror' mode with a dominant $\delta B_{\|}$perturbation when $\omega_{M}{ }^{2} \equiv\left(\sigma k_{\|}{ }^{2}+\tau k_{\phi}{ }^{2}\right) V_{A}{ }^{2}$ is negative, where the $\tau k_{u}{ }^{2}$ term represents the destabilizing free energy associated with the anisotropy in the velocity distribution for $\tau<0$.

In general plasma geometry with magnetic field curvature and pressure gradient, an MHD perturbation has both $\delta \overrightarrow{\mathrm{B}}_{\perp}$ and $\delta \mathrm{B}_{\|}$components. In particular, if $\tau k_{\phi}{ }^{2}$ is large, the stability of the ballooning mode must be determined by including the coupling between $\delta \overrightarrow{\mathrm{B}}_{\perp}$ and $\delta \mathrm{B}_{\|}$components. On the other hand, if $\tau$ is near zero, the coupling between $\delta \overrightarrow{\mathrm{B}}_{\perp}$ and $\delta \mathrm{B}_{\|}$components must also be considered to study the stability of the mirror mode. The solutions of the coupled equations. Eys. (22) and (23), are given by

$$
\omega_{ \pm}^{2}=\left[\Gamma \pm\left(\Gamma^{2}-\Sigma\right)^{1 / 2}\right]\left(V_{A}^{2} / 2\right)
$$

where $\Gamma=\left(\omega_{\mathrm{K}}{ }^{2}+\omega_{\mathrm{M}}{ }^{2}\right) / \mathrm{V}_{\mathrm{A}}{ }^{2}=\tau \mathrm{k}_{\phi}{ }^{2}+2 \sigma \mathrm{k}_{\|}{ }^{2}-\mathrm{k}_{\mathrm{c}} \mathrm{P}_{\|}{ }^{\prime} \sin ^{3} \theta / \mathrm{BB}_{\mathrm{o}}$ and $\Sigma=4\left[\left(\tau \mathrm{k}_{\phi}{ }^{2}+\sigma \mathrm{k}_{\|}{ }^{2}\right)\left(\sigma \mathrm{k}_{\|}{ }^{2}-\right.\right.$ $\left.\kappa_{\mathrm{c}} \mathrm{P}_{\|}{ }^{\prime} \sin ^{3} \theta / \mathrm{BB}_{\mathrm{o}}\right)-\mathrm{k}_{\phi}{ }^{2} \sigma \kappa_{\mathrm{c}} \mathrm{P}_{\perp}{ }^{\prime} \sin ^{3} \theta / \mathrm{BB}_{\mathrm{o}}$ ]. If $\Sigma<0$, there is only one purely growing mode with $\omega^{2}=\omega_{-}{ }^{2}$. If $\Gamma^{2}>\Sigma>0$ and $\Gamma<0$, there are two purely growing modes with $\omega^{2}=\omega_{ \pm}{ }^{2}$. If $\Gamma^{2}>\Sigma>0$ and $\Gamma>0$, there is no unstable mode. If $\Sigma>\Gamma^{2}$, there are two unstable complex frequency modes.

On the outer side of the ring current region $\sigma>0$ and $\kappa_{\mathrm{c}} \mathrm{P}_{\perp}{ }^{\prime}>0$, it is possible that $\Sigma<0$ (equivalently, $\omega_{K}{ }^{2} \omega_{M}{ }^{2}<k_{\phi}^{2} \sigma \kappa_{c} P_{\perp}{ }^{\prime} \sin ^{3} \theta / B_{0}$ ) and there is one purely growing mode. The condition can be satisfied even when both $\omega_{K}{ }^{2}>0$ and $\omega_{M}^{2}>0$, i.e., both the simple ballooning instability condition $\left(\omega_{K}^{2}<0\right)$ and the simple mirror instability condition $\left(\omega_{M}{ }^{2}<0\right)$ are not satisfied. This indicates that coupling between $\delta \overrightarrow{\mathrm{B}}_{\perp}$ and $\delta \mathrm{B}_{\|}$can reduce the instability threshold. If 
both $\omega_{\mathrm{K}}{ }^{2}<0$ and $\omega_{\mathrm{M}}{ }^{2}<0$, then $\Gamma<0$, and the condition to have two purely growing modes is $\Gamma^{2}$ $>\Sigma>0$, or equivalently $\left(\omega_{K}{ }^{2}-\omega_{M}{ }^{2}\right)^{2}>-4 k_{\phi}{ }^{2} \sigma K_{c} P_{\perp}{ }^{\prime} \sin ^{3} \theta / B_{0}$ and $\omega_{K}{ }^{2} \omega_{M}{ }^{2}>$ $\mathrm{k}_{\phi}{ }^{2} \sigma K_{\mathrm{c}} \mathrm{P}_{\perp}{ }^{\prime} \sin ^{3} \theta / \mathrm{BB}_{\mathrm{o}}$. If $\omega_{\mathrm{K}}{ }^{2}>0$ and $\omega_{\mathrm{M}}{ }^{2}>0$, then $\Gamma>0$, and the condition to have (wo) unstable modes with complex frequency is $\Gamma^{2}<\Sigma$, or equivalently $\left(\omega_{K}{ }^{2}-\omega_{M}{ }^{2}\right)^{2}<$ $-4 k_{\phi}^{2} \sigma K_{c} P_{\perp}{ }^{\prime} \sin ^{3} \theta / B_{0}$. This condition cannot be met on the outer side of ring current region since $\sigma K_{c} P_{\perp}{ }^{\prime}>0$. Therefore, coupling between ballooning and mirror modes does not create unstable modes with complex frequency.

On the inner side of the ring current region, typically we have $\sigma>0, \kappa_{\mathrm{c}} \mathrm{P}_{\|}{ }^{\prime}<0$, and $\tau>0$. Thus, $\Gamma>0$ and $\Sigma>0$. Therefore, if $\Gamma^{2}>\Sigma$ there is no unstable mode, and if $\Gamma^{2}<\Sigma$ there are two unstable modes with complex frequencies. Since $\tau \approx 1$ and $\omega_{M}{ }^{2} \gg \omega_{K}{ }^{2}$ usually, we expect $\Gamma^{2}>\Sigma$ and there is no unstable mode on the inner side of the ring current region.

To illustrate the above qualitative features we show in Fig. 1 numerical solutions of the stability boundaries in $\left(\beta_{\|}, T_{\perp} / T_{\|}\right)$space for the outer side of the ring current region with typicial equatorial parameters: $\left(\mathrm{k}_{\|} / \mathrm{k}_{\phi}\right)^{2}=10^{-2},\left(\mathrm{k}_{\|}{ }^{2} \mathrm{~L} / \mathrm{K}_{\mathrm{c}}\right)=-1$, and $\partial \ln \mathrm{P} / \partial \ln \mathrm{L}=-5$. Note that for a dipole field $\kappa_{\mathrm{c}}=-3 / \mathrm{L}$ at equator. In Fig. 1 , there are two curves satisfied by $\Sigma=0$. In the region between these two $\Sigma=0$ curves, $\Sigma<0$, and there is only one purely growing mode. In the region below the lower $\Sigma=0$ curve there is no unstable mode. We note that the condition of $\Sigma=()$ cin be approximately given by $\omega_{B}{ }^{2}=0$. The $\omega_{M}{ }^{2}=0$ and $\Gamma=0$ curves lie in this region and almost coincide because $\omega_{K}{ }^{2} \ll \omega_{M}{ }^{2}$. Below the $\omega_{M}{ }^{2}=0$ and $\Gamma=0$ curves in this region, $\omega_{M}{ }^{2}>0$ and $\Gamma$ $>0$, and the unstable mode is dominantly a ballooning type mode; above the $\omega_{M}{ }^{2}=0$ and $\Gamma=0$ curves, $\omega_{M}{ }^{2}<0$ and $\Gamma<0$, and the unstable mode is mainly a mirror type mode. In the region above the upper $\Sigma=0$ curve, $\Gamma^{2}>\Sigma>0$ and $\Gamma<0$, and there two purely growing modes. Note that the $\omega_{M}{ }^{2}=0$ and $\Gamma=0$ curves are insensitive to the fixed parameters as long as $\left(k_{\|} / k_{0}\right)^{2}<<1$. The $\Sigma=0$ curves are quite sensitive to the fixed parameters; if $\left|k_{\|}{ }^{2} L / k_{c}\right|$ decreases or $|\partial \ln P / \partial \ln \mathrm{L}|$ increases, both the $\Sigma=0$ curves move downward, and vice versa. We emphasize that for usual ring current parameters the lower $\Sigma=0$ curve is lower than the $\omega_{M}{ }^{2}=0$ and $\Gamma=0$ curves, and therefore, the critical beta for ballooning mode is lower than for mirror mode. Near the region between the $\omega_{M}{ }^{2}=0$ and $\Gamma=0$ curves there is a strong coupling between the ballooning and the mirror modes, and the mode changes from a ballooning mode at lower $\beta_{\|}$to a mirror mode ill higher $\beta_{\|}$. 
Near the marginal stability and if $\left|\sigma \mathrm{k}_{\|}{ }^{2}\right| \ll\left|\tau \mathrm{k}_{\phi}{ }^{2}\right|$, we have from Eq. (23) $\delta \mathrm{B}_{\|} \approx \mathrm{k}_{\mathrm{o}} \mathrm{P}_{\perp} \hat{\Phi} /$ $\tau \mathrm{B}^{2}$. The usually quoted ballooning mode stability frequency can be obtained from $\mathrm{E} q$. (22) and is approximately given by

$$
\omega^{2} \approx \omega_{B}^{2} \equiv\left[\sigma k_{\|}^{2}-\kappa_{c}\left(P_{\|}{ }^{\prime}+\sigma P_{\perp}{ }^{\prime} / \tau\right) \sin ^{3} \theta / B_{0}\right] V_{A}^{2}
$$

Similarly, the mirror mode stability frequency is approximately given by

$$
\omega^{2} \approx \omega_{M}^{2} \equiv\left[\sigma \mathrm{k}_{\|}^{2}+\tau \mathrm{k}_{\phi}^{2}\right] \mathrm{V}_{\mathrm{A}}^{2}
$$

Figure 2(a) shows the magnetic latitudinal dependence of $\Omega_{\mathrm{M}}{ }^{2}, \Omega_{\mathrm{B}}{ }^{2}$, and $\Omega_{ \pm}{ }^{2}$ along a dipole field line for $\mathrm{L}=6.6 \mathrm{R}_{\mathrm{E}}, \mathrm{T}_{\perp} / \mathrm{T}_{\|}(\theta=0)=1.5, \beta_{\|}(\theta=0)=0.75, \mathrm{~m}=2\left(\mathrm{)},\left(\mathrm{k}_{\|} \mathrm{L}\right)^{2}=4\right.$, and $\partial \ln P / \partial \operatorname{lnL}=-5$, where $\Omega_{M}{ }^{2}, \Omega_{B}{ }^{2}$, and $\Omega_{ \pm}{ }^{2}$ are normalized in the form of $\left(\omega L / m V_{A}(\theta=())\right)^{2}$ with $\mathrm{m}$ being the azimuthal mode number. Figure $2(\mathrm{~b})$ shows the corresponding $\theta$ dependence of $T_{\perp} / \Gamma_{\|}$ and $\beta_{\|}$along the field line. It is clear that $\Omega_{\mathrm{B}}{ }^{2}$ and $\Omega_{\mathrm{M}}{ }^{2}$ are good approximations of $\Omega_{ \pm}{ }^{2}$. This is because $\tau>0$ along the field line for the set parameters. On the other hand if $\tau=0$ at some $\theta_{0}$. there will be a strong coupling between the mirror and the ballooning modes around $\theta_{0}$ where mode transition occurs. Figure 3(a) shows the magnetic latitudinal dependence of $\Omega_{\mathrm{M}}{ }^{2}, \Omega_{\mathrm{B}}{ }^{2}$, and $\Omega_{ \pm}{ }^{2}$ along the dipole field line for $L=6.6 R_{E}, T_{\perp} / T_{\|}=2.0$ at $\theta=0, \beta_{\|}(\theta=())=1 .(), m=2()$, $\left(k_{\|} L\right)^{2}=4$, and $\partial \ln P / \partial \ln L=-5$. The corresponding $\theta$ dependence of $T_{\perp} / T_{\|}$and $\beta_{\|}$along the tield line is shown in Fig. 3(b). At $\theta_{\mathrm{O}}\left(\approx 9^{\circ}\right)$ where $\tau=0, \Omega_{\mathrm{B}}{ }^{2}$ is discontinuous and changes from $\infty$ (c) $-\infty$. The $\Omega_{+}{ }^{2}$ branch changes from a stable ballooning type $\left(\Omega_{\mathrm{B}}{ }^{2}\right)$ mode in the low latitude region to a stable mirror type $\left(\Omega_{\mathrm{M}}{ }^{2}\right)$ mode in the high latitude region. Similarly, the $\Omega_{-}{ }^{2}$ branch changes from an unstable mirror type mode in the low latitude region $\left(\theta<9^{\circ}\right)$ to an unstable ballooning type mode in the high latitude region $\left(\theta>9^{\circ}\right)$.

\subsection{MHD Eigenmode Stability Theory}

We will consider numerical solutions of the eigenmode equations, Eqs. (14) and (15), b) neglecting the nonadiabatic pressure responses $\delta \hat{\mathrm{p}}_{\perp}$ and $\delta \hat{\mathrm{p}}_{\|}$. Thus, the trapped particle kinetic effects such as particle trapping, wave-particle resonance, and finite ion Larmor radius are ignored. There are many eigenmode solutions for a given set of parameters; there are symmetric and antisymmetric field-aligned eigenmode solutions with respect to the equator. For a parity, there are also many eigenmode solutions with different mode structures; $N=0,2,4$, specify symmetric 
modes with $\mathrm{N}=0,2,4, \ldots$ nodes excluding the boundary points, respectively, and $N=1,3,5, \ldots$ specify antisymmetric modes with $N=1,3,5, \ldots$ nodes, respectively. In general, the most unstable mode has the least number of oscillation node and is the $\mathrm{N}=0$ symmetric mode, and the next most unstable mode is the $\mathrm{N}=1$ antisymmetric mode. In the following we will present the results for the $\mathrm{N}=0$ symmetric mode and the $\mathrm{N}=1$ antisymmetric mode.

To compute the perpendicular perturbed magnetic field components we assume that the azimuthal wavelength is much shorter than the wavelength perpendicular to the magnetic flux surface, i.e. $k_{\phi} \gg k_{\psi}$. Then, the $\nabla \psi$ component can be obtained from the Faraday's law and Ohm's law, $\nabla \times(\vec{\xi} \times \vec{B})=\delta \vec{B}$, where $\vec{\xi} \approx-(i \nabla \hat{\Phi}+\vec{A}) \times \vec{B} / B^{2}$ is the plasma displacement, and $\vec{A}$ is the vector potential. In an axisymmetric magnetosphere we have

$$
\delta \mathrm{B}_{\psi} \equiv \delta \overrightarrow{\mathrm{B}} \bullet \nabla \psi /|\nabla \psi|=\overrightarrow{\mathrm{B}} \bullet \nabla(\vec{\xi} \bullet \nabla \psi) /|\nabla \psi| \approx-\mathrm{i} \overrightarrow{\mathrm{B}} \bullet \nabla\left(\nabla \phi \bullet \nabla \hat{\Phi} /|\nabla \phi|^{2}\right) /|\nabla \psi|, \text { (27) }
$$

where $\phi$ is the azimuthal angle and we have assumed that $\nabla \phi \bullet \nabla \hat{\Phi} \gg>\nabla \cdot \vec{A}$ for high-m modes. The azimuthal component of the perturbed magnetic field is given by

$$
\delta \overrightarrow{\mathrm{B}} \bullet \nabla \phi /|\nabla \phi|=\overrightarrow{\mathrm{B}} \bullet \nabla(\vec{\xi} \bullet \nabla \phi) /|\nabla \phi| \approx \overrightarrow{\mathrm{B}} \bullet \nabla\left[\nabla \psi \bullet \overrightarrow{\mathrm{A}} /|\nabla \psi|^{2}\right] /|\nabla \phi|
$$

Note that $\nabla \psi \bullet \vec{A}$ is larger than $\nabla \psi \bullet \nabla \hat{\Phi}$ and is related to the parallel magnetic field component by $\delta \mathrm{B}_{\|}=\overrightarrow{\mathrm{B}} \bullet \nabla \times \overrightarrow{\mathrm{A}} / \mathrm{B} \approx-\mathrm{B} \partial\left[\nabla \psi \bullet \overrightarrow{\mathrm{A}} /|\nabla \psi|^{2}\right] / \partial \phi$. Thus, if the perturbed quantities are proportional I( $\exp (\operatorname{im} \phi)$, we have

$$
\delta \mathrm{B}_{\phi} \equiv \delta \overrightarrow{\mathrm{B}} \bullet \nabla \phi /|\nabla \phi| \approx \mathrm{i} \overrightarrow{\mathrm{B}} \bullet \nabla\left(\delta \mathrm{B}_{\|} / \mathrm{mB}\right) /|\nabla \phi|
$$

\subsubsection{Symmetric $\delta B_{\|}$Modes}

Figure 4 shows the field-aligned structure of the three perturbed magnetic field components (a) $\delta \mathrm{B}_{\|}$, (b) $\delta \mathrm{B}_{\psi}$, (c) $-\mathrm{i} \delta \mathrm{B}_{\phi}$ normalized by $\mathrm{B}_{0}$, and (d) the normalized electrostatic potential $\delta \Phi$ $\left(=\Phi / \omega L^{2} B_{0}\right.$, where $\Phi$ is the electrostatic potential) of a marginally stable $N=()$ symmetric ballooning mode for $\mathrm{L}=6.6 \mathrm{R}_{\mathrm{E}}, \mathrm{T}_{\perp} / \mathrm{T}_{\|}=1.5$ at $\theta=0, \beta_{\|}(\theta=0)=0.10068$ ( $\tau=0.928$ ). azimuthal mode number $\mathrm{m}=50$, and $\partial \ln \mathrm{P} / \partial \ln \mathrm{L}=-5$. We note that the radial magnetic field $\delta B_{\psi}$ is the dominant component. Figure 5(a) shows the marginal stability boundaries of the $N=0$ symmetric ballooning modes in the equatorial $\left(\beta_{\|}, T_{\perp} / T_{\|}\right)$space for $\partial \ln \mathrm{P} / \partial \ln \mathrm{L}=-5,-2.5$, anl -1 
with the fixed parameters: $L=6.6 R_{E}$ and azimuthal mode number $m=50$. Also shown in Fig. $5(a)$ is the $\tau=0$ curve above which the mirror instability criterion is satisfied locally. I is clear thal the $\mathrm{N}=0$ symmetric ballooning mode has a small critical beta that is much smaller than the locial mirror stability threshold. The pressure anisotropy is destabilizing and can lower the critical betia for ballooning mode. We note that the critical beta value for the $\mathrm{N}=0$ symmetric ballooning mode for $\partial \ln \mathrm{P} / \partial \ln \mathrm{L}=-5$ is about the same as the local theory prediction presented in Fig. 1. The small discrepancy is mainly due to the use of fixed values of $\left(k_{\|} / k_{\phi}\right)^{2}$ and $\left(k_{\|}{ }^{2} L / k_{c}\right)$ in the local theory calculation. The eigenmode solutions provide the variation of $\left(k_{\|} / k_{\phi}\right)^{2}$ and $\left(k_{\|}{ }^{2} L / k_{c}\right)$ along the field line. The corresponding stability boundaries in the equatorial $\left(\alpha_{p}, \tau\right)$ space are also shown in Fig. 5(b), where $\alpha_{p}$ is defined in Eq. (19). Above the marginal stability boundary curves the $\dot{N}=()$ symmetric ballooning modes are unstable. We note that the stability curves are much closer to eilch other (within $50 \%$ ) in the equatorial $\left(\alpha_{p}, \tau\right)$ space than in the equatorial $\left(\beta_{\|}, T_{\perp} / T_{\|}\right)$space. It is important to point out that the critical beta or $\alpha_{p}$ thresholds for the $N=0$ MHD ballooning modes are much smaller than those for the $\mathrm{N}=1$ modes presented in Sec. 3.2.2

An important comment is made here that the $\mathrm{N}=0$ modes will be stabilized if the kinetic effects associated with the energetic trapped particles are included. This will be discussed in Sec: 3.3. On the other hand, the kinetic trapped particle effects on the stability of the $N=1$ mode are relatively small. Therefore, the $N=1$ MHD mode has the lowest critical beta threshold for instability.

\subsubsection{Antisymmetric $\delta B_{\|}$Modes}

Figure 6 shows (a) the field-aligned structure of the three perturbed magnetic field components $\delta \mathrm{B}_{\|}, \delta \mathrm{B}_{\psi}$, and $-\mathrm{i} \delta \mathrm{B}_{\phi}$ normalized by $\mathrm{B}_{0}$, and (d) the normalized electrostitic potential $\delta \Phi\left(=\Phi / \omega L^{2} B_{0}\right.$, where $\Phi$ is the electrostatic potential) of a marginally stable $\mathrm{N}=1$ intisymmetric ballooning mode for $L=6.6 R_{E}, T_{\perp} / T_{\|}(\theta=0)=1.6, \beta_{\|}(\theta=0)=1.179(\tau=-0.1317)$, azimuthu! mode number $\mathrm{m}=50$, and $\partial \ln \mathrm{P} / \partial \operatorname{lnL}=-2$. Note that $\delta \mathrm{B}_{\|}$is antisymmetric with respect the equator and is localized with a latitudinal spread of about $20^{\circ}$. The transverse magnelic components, radial component $\delta \mathrm{B}_{\psi}$ and azimuthal component $\delta \mathrm{B}_{\phi}$, have a symmetric parity and their polarization varies with the magnetic latitude. From Eq. (29) we see that the azimuthal component $\delta \mathrm{B}_{\phi}$ is proportional to the field-aligned derivative of $\delta \mathrm{B}_{\|}$, but is inversely proportional to the azimuthal mode number. We also note that the field-aligned wave structure is similar 10 those of the multisatellite ( SCATHA, GOES2, GOES3, GEOS2 ) observations of a long lasting compressional Pc 5 wave event during November 14-15, 1979 |Takahashi et al., 1987; 'Takilhishi. 
et al., 1990] which revealed the field-aligned structures of the compressional magnetic field $\delta B_{\|}$(6) be antisymmetric with respect to the earth's magnetic equator with a full latitudinal range of about $20^{\circ}$.

From Fig. (6) the maximum value of the normalized electrostatic potential of the ballooning-mirror mode is roughly $\delta \Phi_{\max } \approx 10^{-3}\left(\delta \mathrm{B}_{\|} / \mathrm{B}_{0}\right)_{\max }$, and the maximum azimuthal electric field in the ring current region can be estimated. Assuming that $\left(\delta \mathrm{B}_{\|} / \mathrm{B}_{0}\right)_{\max }=0.05$, a typical hot proton energy $T_{h} \approx 10 \mathrm{keV}$, a hot proton Larmor radius $\rho_{L} \approx 100 \mathrm{~km}$ for an equatorial magnetic field with $B_{0} \approx 100 \mathrm{nT}$, and the wave frequency is much smaller th:n the proton cyclotron frequency with $\left(\omega / \omega_{c}\right) \approx 10^{-2}$, the maximum perpendicular electric field near the equatorial plane is estimated to be $\delta \mathrm{E}_{\phi_{\max }} \approx \mathrm{m} \Phi_{\max } / \mathrm{L}=\left(2 \omega / \omega_{\mathrm{c}}\right)\left(\mathrm{mL} / \mathrm{\rho}_{\mathrm{L}}\right)\left(\mathrm{T}_{\mathrm{h}} / \mathrm{e} \mathrm{p}_{\mathrm{L}}\right) \delta \Phi_{\max } \approx 2 \mathrm{mV} / \mathrm{m}$, which is aboul the same order as the typical dawn-dust electric field. Therefore, energetic particles resonating in phase with the ULF wave can be accelerated (or decelerated) in the azimuthal direction and will gain (or lose) about $13 \mathrm{keV}$ in a traveling distance of one Earth radius.

The corresponding perturbed current density can be obtained from $\delta \vec{j} \approx i \vec{k} \times \delta \vec{B}$, where ik $=(\vec{B} / B) \bullet \nabla$. The three components of the perturbed current density is given by the radial component $\delta \mathrm{J}_{\psi}=\mathrm{i}\left(\mathrm{k}_{\phi} \delta \mathrm{B}_{\|}-\mathrm{k}_{\|} \delta \mathrm{B}_{\phi}\right)$, the azimuthal component $\delta \mathrm{J}_{\phi}=\mathrm{i}\left(\mathrm{k}_{\|} \delta \mathrm{B}_{\psi}-\mathrm{k}_{\psi} \delta \mathrm{B}_{\|}\right)$, ind the fieldaligned component $\delta J_{\|}=i\left(k_{\psi} \delta B_{\phi}-k_{\phi} \delta B_{\psi}\right)$. Figures 7 shows the three components of the perturbed current density, (a) $-i \delta J_{\|}$, (b) $-i \delta J_{\psi}$, and (c) $\delta J_{\phi}$, normalized by $\left(B_{0} / L\right)$ for the corresponding magnetic field perturbation shown in Fig. 6. It is clear that the field-aligned currem density is the dominant component near the ionosphere-magnetosphere boundary. Assuming thill $\left(\delta \mathrm{B}_{\|} / \mathrm{B}_{0}\right)_{\max }=0.05$, the field-aligned current density near the ionosphere-magnetosphere boundar: can be estimated from Fig. 7(a) to be $\delta J_{\|}=10^{-7} \mathrm{~A} / \mathrm{m}^{2}$, which is about 20 times larger than the typical westward ring current density of $J_{\phi} \approx 5 \times 10^{-9} \mathrm{~A} / \mathrm{m}^{2}$. The large field-aligned currem density generated near the ionosphere-magnetosphere boundary by the ballooning-mirror instibilit! may be related to the substorm activities.

Figure 8(a) shows the marginal stability boundaries of the $\mathrm{N}=1$ antisymmetric mode in the equatorial $\left(\beta_{\|}, \mathrm{T}_{\perp} / \mathrm{T}_{\|}\right)$space for $\partial \ln \mathrm{P} / \partial \ln \mathrm{L}=-100,-50,-25,-10,-5,-2.5$, and -1 , respectivel! . with the fixed parameters: $\mathrm{L}=6.6 \mathrm{R}_{\mathrm{E}}$ and azimuthal mode number $\mathrm{m}=50$. Also shown in Fig. 8 (a) is the equatorial $\tau=0$ curve above which the mirror instability criterion is satisfied at equator: The pressure anisotropy is destabilizing and can lower the critical beta for ballooning mode presented in Fig. 5(a). We note that the critical beta for the $\mathrm{N}=1$ antisymmetric mode is much higher than the $\mathrm{N}=0$ symmetric ballooning mode. For $T_{\perp} / T_{\|}>1.6($ with $\partial \ln \mathrm{P} / \partial \ln \mathrm{L}=-5$ ) the critical beta is larger than the beta values that satisfy $\tau=0$. Therefore, for equatorial $\left(\beta_{\|}, T_{,} / T_{\|}\right)$ 
values that are above the $\tau=0$ curve, the mode will have mirror mode characteristics and can be classified as mirror mode. The above demonstration clearly shows the transition from an antisymmetric ballooning mode in low $\beta_{\|}$and $T_{\perp} / T_{\|}$to an antisymmetric, dominant mirror-type mode as $\beta_{\|}$and $T_{\perp} / T_{\|}$increase. The corresponding stability boundaries are also shown in Fig. $8(b)$ in the equatorial $\left(\alpha_{p}, \tau\right)$ space. Note that for $\tau<0$, the $\alpha_{p}$ threshold is also negative, and we have plotted absolute value of $\alpha_{p}$ in Fig. $8(\mathrm{~b})$ for $\tau<0$. Above the marginal stability boundary curves the $\mathrm{N}=1$ antisymmetric ballooning-mirror modes are unstable. The marginal stability boundary curves in the equatorial $\left(\alpha_{p}, \tau\right)$ space is very close to each other even for $l()()>$ $|\partial \ln P / \partial \ln L|>1$. This probably holds even for different type of particle velocity distributions. .This property is useful when we compare theoretical calculations with satellite observations where information on $\partial \ln \mathrm{P} / \partial \ln \mathrm{L}$ and particle velocity distribution is either unavailable or can not be obtained accurately.

The field-aligned structure of the perturbed magnetic field of the $N=1$ antisymmentic moskc changes as the mode changes from a ballooning mode in low $\beta_{\|}$and $T_{\perp} / T_{\|}$to a mixed mirrorballooning type mode as $\beta_{\|}$and $T_{\perp} / T_{\|}$increase (or equivalently, as $\tau$ decreases). Figures $\varphi(a)$ - (d) show the field-aligned perturbed magnetic field structures of the marginally stable $N=1$ antisymmetric modes along a marginal stability curve shown in Fig. 8(a) with equatorial values ol (a) $\mathrm{T}_{\perp} / \mathrm{T}_{\|}=1, \beta_{\|}=1.93(\tau=1)$, (b) $\mathrm{T}_{\perp} / \mathrm{T}_{\|}=1.3, \beta_{\|}=1.489(\tau=0.419)$, (c) $\mathrm{T}_{\perp} / \mathrm{T}_{\|}=1$.6. $\beta_{\|}=$ $0.987(\tau=0.052)$, and (d) $T_{\perp} / T_{\|}=2, \beta_{\|}=0.575(\tau=-0.149)$. The fixed parameters are $L=$ 6.6 , azimuthal mode number $\mathrm{m}=50$, and $\partial \ln \mathrm{P} / \partial \ln \mathrm{L}=-5$. For positive values of $\tau$, the locill dispersion indicates a ballooning mode along the field line. As $\tau$ decreases, the amplitudes of the parallel magnetic field component $\delta B_{\|}$and the azimuthal component $\delta B_{\phi}$ increase, and the mode also becomes more localized. As $\tau$ becomes negative, the local dispersion is a mirror mode neall the equator, but has ballooning mode characteristics as the latitude increases away from the equatorial region. In fact, Figs. 9(c) and 9(d) resemble the field-aligned wave structures (all threc perturbed magnetic field components) of the multisatellite ( SCATHA, GOES2, GOES3, GEOS2, observations of a long lasting compressional Pc 5 wave event during November 14-15, 1974 [Takahashi et al., 1987; Takahashi, et al., 1990]. This strongly suggests that this particulill multisatellite observation may be related to the marginally stable ballooning-mirror mode with ? value close to zero.

It is interesting to point out that the perturbed magnetic field near the ionosphere boundiary is dominated by the radial component for the internally excited ballooning-mirror mode. On the other hand, for the field line resonance (external excitation) the perturbed magnetic field near the 
ionosphere boundary is dominated by the azimuthal component [Cheng et al., 1993al. This knowledge can be used to help determine the excitation mechanism of ULF waves.

\subsection{Trapped Particle Stabilization of Symmetric Ballooning Modes}

From the MHD eigenmode calculations we have found that the symmetric ballooning modes have lower critical beta thresholds than the antisymmetric ballooning-mirror modes. Therefore, from the MHD theory one would conclude that modes with symmetric $\delta \mathrm{B}_{\|}$field-aligned structure should be observed. However, the satellite observations clearly indicate that compressional Pc 5 waves have antisymmetric $\delta B_{\|}$field-aligned structure. This paradox can be resolved if the kinetic trapped particle effect is retained in the analysis. In fact, numericul solution. including the kinetic trapped particle effect show that ballooning modes with symmetric $\delta B_{\|}$fieldaligned structure are stable for the parameters of interest in the ring current region. In the following, we will perform an analytical analysis to support the conclusion.

Considering modes near marginal stability and assuming that $\left|\sigma \mathrm{k}_{\|}{ }^{2}\right| \ll<\left|\tau k_{0}{ }^{2}\right|$, then from Eq. (15), we have $\delta \mathrm{B}_{\|}-\left(\overrightarrow{\mathrm{B}} \times \tilde{\nabla} \mathrm{P}_{\perp} \bullet \nabla \hat{\Phi} / \mathrm{B}^{2}+\delta \hat{\mathrm{p}} \perp\right) / \tau \mathrm{B}$. Then Eq. (14) reduces to

$$
\begin{aligned}
\vec{B} & \nabla\left(\frac{\sigma k_{\perp}^{2}}{B^{2}} \vec{B} \cdot \nabla \hat{\Phi}\right)+\frac{\rho k_{\perp}^{2}}{B^{2}} \hat{\Phi} \\
& +\frac{(\vec{B} \times \vec{K})}{B^{2}} \bullet \vec{k}_{\perp}\left[\left(\frac{\vec{B} \times \tilde{\nabla} P_{\|}}{B^{2}}+\frac{\sigma \vec{B} \times \tilde{\nabla} P_{\perp}}{\tau B^{2}}\right) \cdot \vec{k}_{\perp} \hat{\Phi}+\delta \hat{p}_{\|}+\sigma \delta \hat{p}_{\perp} / \tau\right]=0 .
\end{aligned}
$$

In the limit of small gyroradius and small frequency, we can neglect contributions that ure on the order of $\left(\omega / \omega_{b}\right), \omega_{b}$ is the trapped bounce frequency. The nonadiabatic trapped particle pressurc perturbation is then given by [Cheng and Lin, 1987]

$$
\left(\begin{array}{c}
\delta \hat{p}_{\| 1} \\
\delta \hat{p}_{\perp}
\end{array}\right)=\sum_{j} \int d^{3} v \frac{\left(-\omega \partial F / \partial \varepsilon-\omega_{*} F / \varepsilon_{0}\right)}{\left(\omega-\left\langle\omega_{d}\right\rangle\right)}<Z>\left(\begin{array}{c}
2(\varepsilon-\mu B) \\
\mu B
\end{array}\right)+O\left(\omega / \omega_{h}\right),
$$

where $Z=e \omega_{d} \hat{\Phi}+M \mu \delta B_{\|}, e$ is the particle charge, the trapped particle bounce average of $Z$ is given by $\left\langle Z>=\oint d s Z A_{\|}\left|/ \oint d s / v_{\|}\right|\right.$where the integration is along the trapped particle trajectory in 
one bounce period, $\omega_{4}=\mathrm{cM} \varepsilon_{0}\left(\vec{B} \times \nabla \ln F \bullet \vec{k}_{\perp}\right) / e B^{2}, M \varepsilon_{0}$ is the typical particle energy, and $M$ is the particle mass. To carry out the velocity integration in Eq. (31), we further assume $\omega \ll \omega_{*}$. $\left\langle\omega_{d}\right\rangle$ and $\left.\langle Z\rangle \approx e<\omega_{d}\right\rangle \hat{\Phi}$. These approximations are reasonably good because the $\delta B_{\|}$term is small in comparison with the potential term in $\mathrm{Z}$, and near the marginal stability the mude frequency is much smaller than the diamagnetic drift and magnetic drift frequencies of the hol trapped particles. Equation (31) can be integrated to give

$$
\left(\frac{\overrightarrow{\mathrm{B}} \times \tilde{\nabla} \mathrm{P}_{\|}}{\mathrm{B}^{2}}+\frac{\sigma \overrightarrow{\mathrm{B}} \times \tilde{\nabla} \mathrm{P}_{\perp}}{\tau \mathrm{B}^{2}}\right) \cdot \overrightarrow{\mathrm{k}}_{\perp} \hat{\Phi}+\delta \hat{\mathrm{p}}_{\|}+\sigma \delta \hat{\mathrm{p}}_{\perp} / \tau \approx 0
$$

Thus, Eq. (32) shows that the fluid magnetic field curvature-pressure gradient driven term in the ballooning mode equation, Eq. (30), is canceled by the nonadiabatic hot trapped particle pressure response. Therefore, Eq. (30) only admits stable symmetric $\hat{\Phi}$ and $\delta B_{\|}$Alfvén eigenmodes. Physically, the hot trapped ion stabilization of ultra low frequency $\left(\omega \ll \omega_{*},<\omega_{d}>\right)$ MHD mucles with symmetric $\hat{\Phi}$ and $\delta B_{\|}$structure is due to the fact that the hot trapped ion dynamic is no longer governed by the $E \times \vec{B}$ drift, but rather by the magnetic $\nabla B$ and curvature drifts. To perturb the hos trapped ion pressure the hot ion precession orbit must be perturbed through the perturbed electric and magnetic fields. Since the hot trapped ions precess very rapidly across the $\vec{B}$ field, the motion becomes very rigid with respect to the ultra low frequency MHD perturbation. Equition (32) indicates that the nonadiabatic hot trapped ion pressure changes through the perturbed perpendicular magnetic field (through electrostatic potential $\Phi$ ) so that their total pressure perturbation due to both the fluid and kinetic responses is zero (see Eq. (11) in the limit of smilll $\left.\delta B_{\|}\right)$.

Again, we emphasize that the nonadiabatic perturbed hot trapped ion pressure responding to low frequency antisymmetric $\hat{\Phi}$ and $\delta B_{\|}$perturbations is vanishingly small becaluse the bounce average of perturbed quantities is zero. This is because that the hot ions bounce very rapidly allony the north-south symmetric field line and the nonadiabatic hot ion pressure response from perturbations in the northern hemisphere cancels that from the southern hemisphere. Thus the hol ion fluid pressure response survives and determines the ballooning-mirror stability. Therefore, the antisymmetric ballooning-mirror modes have the lowest critical beta threshold for instability, and we expect that the ULF waves observed in the ring current region should have antisymmetric $\Phi$ and $\delta \mathrm{B}_{\|}$field-aligned structures. 


\section{Summary and Discussion}

In the paper we have performed a kinetic-MHD eigenmode stability study of the ballooning-mirror mode for anisotropic pressure plasmas in a dipole magnetic field. We consider that $\left(\omega-\omega_{d}\right) \ll \omega_{b}$ and neglect small kinetic contributions in the nonadiabatic pressure responses: $\delta \hat{\mathrm{p}}_{\perp}$ and $\delta \hat{\mathrm{p}}_{\|}$that are in the order of $\left(\omega / \omega_{\mathrm{b}}\right)$. The eigenmode equations take into account the coupling of the transverse and compressional components of the perturbed magnetic field. By totally neglecting $\delta \hat{\mathrm{p}}_{\perp}$ and $\delta \hat{\mathrm{p}}_{\|}$, the eigenmode equations are essentially MHD equations with anisotropic pressure along the ambient magnetic field line. It is shown that the MHD ballooning modes with symmetric $\delta \Phi$ and $\delta B_{\|}$structures along the north-south equilibrium magnetic field lines have the lowest beta stability threshold. The antisymmetric MHD modes have higher belil stability threshold. For small pressure anisotropy the antisymmetric MHD mode is a pure ballooning mode. But, for higher pressure anisotropy with simple mirror threshold ( $\tau=()$ ) exceeded at equator, the antisymmetric MHD mode becomes a hybrid mirror-ballooning mode with strong coupling between the transverse and the parallel magnetic field compunents. When the leading kinetic contribution from $\delta \hat{\mathrm{p}}_{\perp}$ and $\delta \hat{\mathrm{p}}_{\|}$is included in the analysis for the symmetric modes, the symmetric ballooning modes are shown both analytically and numerically to be stabilized by the energetic trapped particle kinetic effect in the absence of wave-particle resonance. The stabilization results from the fact that the hot trapped ions precess very rapidly across the $\vec{B}$ field, their motion becomes very rigid with respect to low frequency MHD perturbations with symmetric structure of parallel perturbed magnetic field $\delta \mathrm{B}_{\|}$and electrostatic potential $\Phi$ along the north-south ambiem magnetic field, and thus they do not contribute to the destabilizing pressure gradient to drive ballooning iristability. On the other hand, the energetic trapped ion's rapid bounce motion tends w average out the nonadiabatic pressure contributions associated with the antisymmetric $\delta \mathrm{B}_{\|}$and $\widehat{\Phi}$ wave structure along the north-south ambient magnetic field. Therefore, the antisymmetric ballooning-mirror mode is only weakly influenced by energetic trapped particle kinetic effects and has the lowest critical beta threshold mainly determined by MHD theory.

Based on a local bi-Maxwellian velocity distribution, the stability of the ballooning-mirror mode is numerically computed and is expressed in terms of parallel plasma beta $\beta_{\|}$and temperature ratio $T_{\perp} / T_{\|}$at equator. The field-aligned structure of the perturbed magnetic field of the $N=1$ antisymmetric mode changes from a pure ballooning mode with dominant transverse magnetic field component at small values of $\beta_{\|}$and $T_{\perp} / T_{\|}$to a mixed mirror-ballooning type mode with comparable transverse and compressional magnetic field components near the equator as $\beta_{\|}$and 
$\mathrm{T}_{\perp} / \mathrm{T}_{\|}$increase so that $\tau<0$. As $\tau$ decreases, the amplitudes of the parallel magnetic field component $\delta \mathrm{B}_{\|}$and the azimuthal component $\delta \mathrm{B}_{\phi}$ increase, and the mode also becomes more localized near the equator. In fact, at negative or small positive values of $\tau$ the theoreticill eigenmode structures resemble the field-aligned wave structures (all three perturbed magnetic field components) of the multisatellite (SCATHA, GOES2, GOES3, GEOS2) observations of a long lasting compressional Pc 5 wave event during November 14-15, 1979 [Takahashi et al., 1987: Takahashi, et al., 1990]. This strongly suggests that this particular multisatellite observation maly be related to the marginally stable ballooning-mirror mode with $\tau$ value close to zero.

The electric field and field-aligned current associated with the ballooning-mirror instability can be evaluated. Assuming $\left(\delta \mathrm{B}_{\mid} / \mathrm{B}_{0}\right)_{\max }=0.05$, the maximum perpendicular electric field neill the equatorial plane is about a few $\mathrm{mV} / \mathrm{m}$ in the ring current region, and is the same order of magnitude as the typical dawn-dust electric field. Near the ionosphere-magnetosphere boundary the corresponding perturbed field-aligned current density is estimated to be one to two order of magnitude larger than the typical westward ring current density. Thus, large field-aligned currem density generated near the ionosphere-magnetosphere boundary by the ballooning-mirror instibilit! may be related to the substorm activities.

The stability of the ballooning-mirror mode can be better given in terms of the equatorial values of $\alpha_{p}$ and $\tau$, where $\alpha_{p}$ represents the product of pressure gradient and magnetic field curvature and is defined in Eq. (19), and $\tau$ represents the combined effect of plasima betal and pressure anisotropy and is defined in Eq. (7). The marginal stability boundaries in the equatorial $\left(\beta_{\|}, T_{\perp} / T_{\|}\right)$space are very sensitive to the pressure gradient. But the marginal stability boundiry curves in the equatorial $\left(\alpha_{p}, \tau\right)$ space is very close to each other even for $10>|\partial \ln P / \partial \ln L|>() .1$. This probably holds even for different type of particle velocity distributions. The property shoulu be useful when we compare theoretical calculations with those obtained from satellite observitions. where information on $\partial \ln \mathrm{P} / \partial \operatorname{lnL}$ is either unavailable or can not be obtained accurately.

Although significant progress has been made in the satellite observational data of $\mathrm{PC}+-5$ waves and theoretical understandings of these waves, a close comparison between theory ind observation is still required to provide better understanding of the plasma and wave properties. $A$ comprehensive theory not only helps to explain the observations but also provides new walys of measuring plasma properties and wave structures. However, a more detailed measurement of energetic particle energy and pitch angle distributions during ULF wave events would be repuired to give a conclusive test of the instability threshold of the ballooning-mirror instabilities. A correlation study between the instability parameters $\alpha_{p}$ and $\tau$ obtained from satellite particle ditil 
during Pc 4-5 wave events and the theoretical ballooning-mirror instability threshold conditions has recently been performed [Cheng, et al., 1993b]. The results clearly suggest that the transverse wave is related to the ballooning mode at lower beta. The compressional wave is related to a mixed ballooning-mirror mode excited when pressure gradient and pressure anisotropy $\left(P_{\perp} / P_{\|}\right)$are large.

In this paper we have neglected wave-particle esonance. To consider wave-particle resonance one needs to include both hot and core plasma contributions. Typically, the hot particles resonating with the wave will give a destabilizing effect to the ballooning-mirror stability because $\omega$ $<\omega_{* h}$. On the other hand $\omega>\omega_{* c}$, and the core (cold) plasma resonating with the wave will have a stabilizing effect to the ballooning-mirror stability. The net effect of the wave-particle resonance due to all particle species will probably modify the stability threshold slightly from those computed in this paper. If the wave-particle resonance destabilizes the ballooning-mirror mode at plasma beta below the MHD critical beta, the resultant growth rate will be very weak compared with the MHD growth rate. Similar results of resonance effects on ballooning mode have been studied for toroidal plasmas [Cheng, 1982a,b].

It is also important to note that the theory of ballooning-mirror instabilities presented in the paper have zero real frequency when the ballooning-mirror modes are unstable. The real frequency of the ballooning-mirror instabilities must be determined by considering the Doppler shift frequency associated $w^{i} \mathrm{~h}$ the balk plasma convection as well as the diamagnetic and magnetic drift frequencies of various plasma species. As long as the mode frequency, the core plasma diamagnetic drift frequencies, and the Doppler shift frequency associated with the balk plasma convection are all small compared with the hot ion magnetic drift and diamagnetic drift frequencies, the analysis performed in the paper is valid in the lowest order in the frequency ordering. Finally, we note that the theoretical investigation can still be improved by employing self-consistent magnetospheric equilibrium [Cheng, 1992] for more accurate stability threshold calculation. These effects on the stability of ballooning-mirror mode will be investigated in the future.

\section{Acknowledgments}

This work is supported by the U.S. National Science Foundation Grant No. ATM9217859 and U. S. Departmert of Energy Contract No. DE-AC02-76-CHO3073 at Princeton University. 


\section{References}

Allan, W., E. M. Poulter, and E. Nielsen, STARE observations of a Pc 5 pulsation with large azimuthal wave number, J. Geophys. Res., 87, 6163, 1982.

Chen, L., and A. Hasegawa, Kinetic theory of geomagnetic pulsations 1. internal excitations by energetic particles, J. Geophys. Res., 96, 1503, 1991.

Cheng, C. Z., Kinetic theory of collisionless ballooning modes, Phys. Fluids, 25, 1020, 1982 al.

Cheng, C. Z., High-n collisionless ballooning modes in axisymmetric toroidal plasmas, Nucl. Fusion, 22, 773, $1982 \mathrm{~b}$.

Cheng, C. Z. and C. S. Lin, Eigenmode analysis of compressional waves in the magnetosphert. Geophys. Res. Lett., 14, 884, 1987.

Cheng, C. Z., A kinetic-MHD model for low frequency phenomena, J. Geophys. Res., 96. $21159,1991$.

Cheng, C. Z., Magnetospheric equilibrium with anisotropic pressure, J. Geophys. Res., 97. 1497, 1992.

Cheng, C. Z., T. C. Chang, C. A. Lin, and W. H. Tsai, Magnetohydrodynamic theory of field line resonance in the magnetosphere, J. Geophys. Res., 98, 11,339, 1993a.

Cheng, C. Z., K. Takahashi, and A. T. Y. Lui, Correlation between ballooning-mirror instabilit! and ULF wave events, to be submitted to J. Geophys. Res., 1993b.

Engebretson, M. J., L. J. Zanetti, T. A. Potemra, D. M. Klumpar, R. J. Strangeway, and M. H. Acuna, Observations of intense ULF pulsation activity near the geomagnetic equator during quiet times, J. Geophys. Res., 93, 12795, 1988.

Grad, H., The guiding center plasma, in Magneto-Fluid and Plasma Dynamics, Symp. in Appl. Math., Vol. 18, p. 162, American mathematical Society, New York, 1967.

Hasegawa, A., Drift mirror instabilities in the magnetosphere, Phys. Fluids, 12, 2642, 1969.

Hasegawa, A., Drift wave instabilities of a compressional mode in a high b plasma, Phys. Res. Lett., 27, 11, 1971.

Higuchi, T., S. Kokubun, and S. Ohtani, Harmonic structure of compression at synchronous: orbit, Geophys. Res. Lett., 13, 1101, 1986.

Judge, D. L., and P. J. Coleman, Jr., Observations of low-frequency hydromagnetic waves in the distant geomagnetic field, Explorer 6, J. Geophys. Res., 67, 5071, 1962.

Lin, C. S., and J. N. Barfield, Azimuthal propagation of storm time Pc 5 waves observed simultaneously by geostationary satellites GOES2 and GOES3, J. Geophys. Res., 9(). $11075,1985$. 
Misra, K. D., E. Nielsen, and L. Luhr, STARE and SABRE observations of a Pc 5 with liurge azimuthal wave number during a storm period, J. Geophys. Res., 90, 10923, 1985.

Nagano, H., and T. Araki, Long-duration Pc 5 pulsations observed by geostationary satellites. Geophys. Res. Lett., 10, 908, 1983.

Ng, P. H., V. L. Patel, and S. Chen, Drift compressional instability in the magnetosphere, J. Geophys. Res., 89, 10763, 1984.

Pokhotelov, O. A., V. A. Pilipenko, Yu. M. Nezlina, J. Woch, G. Kremser, A. Korth, and E. Amata, Excitation of high- $\beta$ plasma instabilities at the geostationary orbit; theory and observations, Planet. Space Sci., 34, 695, 1986.

Southwood, D. J., A general approach to low-frequency instability in the ring current plasma, J. Geophys. Res., 81, 3340, 1976.

Takahashi, K., P. R. Higbie, and D. N. Baker, Azimuthal propagation and frequency characteristic of compressional Pc 5 waves observed at geostationary orbit, J. Geophy's Res., 90, 1473, 1985a.

Takahashi, K., C.T. Russell, and R. R. Anderson, ISEE 1 and 2 observation of the spatial structure of a compressional Pc 5 wave, Geophys. Res. Lett., 12, 613, 1985 b.

Takahashi, K., J. F. Fennell, E. Amata, and P. R. Higbie, Field-aligned structure of the storm time Pc 5 waves of November 14-15, 1979, J. Geophys. Res., 92, 5857, 1987.

Takahashi, K., Multisatellite studies of ULF waves, Advance in Space Res., 8, 427, 1988.

Takahashi, K., C. Z. Cheng, R. W. McEntire, and L. M. Kistler, Observation and theory of Pc 5 waves with harmonically related transverse and compressional components, J. Geophys. Res., 95, 977, 1990.

Walker, A. D. M., R. A. Greenwald, A. Korth, and G. Kremser, STARE and GEOS2 observations of a storm time Pc 5 ULF pulsation, J. Geophys. Res., 87, 9135, 1982. 


\section{Figure Captions}

Fig. 1. Local stability boundaries in $\left(\beta_{\|}, T_{\perp} / T_{\|}\right)$space for the outer side of the ring current region with typical equatorial parameters: $\left(\mathrm{k}_{\|} / \mathrm{k}_{\phi}\right)^{2}=10^{-2},\left(\mathrm{k}_{\|}{ }^{2} \mathrm{~L} / \mathrm{K}_{\mathrm{c}}\right)=-1$, and $\partial \ln \mathrm{P} / \partial \ln \mathrm{L}=-5$. Note that for a dipole field $\kappa_{c}=-3 / \mathrm{L}$ at equator.

Fig. 2. (a) The magnetic latitudinal dependence of $\Omega_{\mathrm{M}}{ }^{2}, \Omega_{\mathrm{B}}{ }^{2}$, and $\Omega_{ \pm}{ }^{2}$ along the dipole field line for $\mathrm{L}=6.6 \mathrm{R}_{\mathrm{E}}, \mathrm{T}_{\perp} / \mathrm{T}_{\|}(\theta=0)=1.5, \beta_{\|}(\theta=0)=0.75, \mathrm{~m}=20,\left(\mathrm{k}_{\|} \mathrm{L}\right)^{2}=4$, and $\partial \ln \mathrm{P} / \partial \ln \mathrm{L}=$ -5 , where $\Omega$ is the normalized frequency given by $\left(\omega \mathrm{L} / \mathrm{mV}_{\mathrm{A}}(\theta=0)\right)$ with $\mathrm{m}$ being the azimuthal mode number. (b) The corresponding $\theta$ dependence of $T_{\perp} / T_{\|}$and $\beta_{\|}$along the field line.

Fig. 3. (a) The magnetic latitudinal dependence of $\Omega_{\mathrm{M}}{ }^{2}, \Omega_{\mathrm{B}}{ }^{2}$, and $\Omega_{ \pm}{ }^{2}$ along the dipole field line for $\mathrm{L}=6.6 \mathrm{R}_{\mathrm{E}}, \mathrm{T}_{\perp} \mathrm{T}_{\|}=2.0$ at $\theta=0, \beta_{\|}(\theta=0)=1.0, \mathrm{~m}=20,\left(\mathrm{k}_{\|} \mathrm{L}\right)^{2}=4$, and $\partial \ln \mathrm{P} / \partial \ln \mathrm{L}$ $=-5$, where $\Omega$ is the normalized frequency given by $\left(\omega \mathrm{L} / \mathrm{mV}_{\mathrm{A}}(\theta=0)\right)$ with $\mathrm{m}$ being the azimuthal mode number. (b) The corresponding $\theta$ dependence of $T_{\perp} / T_{\|}$and $\beta_{\|}$along the field line.

Fig. 4 The field-aligned structure of the three perturbed magnetric field components (a) $\delta B_{\|}$, (b) $\delta \mathrm{B}_{\psi},(\mathrm{c})-\mathrm{i} \delta \mathrm{B}_{\phi}$ normalized by $\mathrm{B}_{0}$, and (d) the normalized electrostatic potential $\delta \Phi$ $\left(=\Phi / \omega L^{2} B_{0}\right.$, where $\Phi$ is the electrostatic potential) of a marginally stable $N=0$ symmetric ballooning mode for $L=6.6 R_{E}, T_{\perp} / T_{\|}=1.5$ at $\theta=0, \beta_{\|}(\theta=0)=0.10068(\tau=0.928)$, azimuthal mode number $\mathrm{m}=50$, and $\partial \ln \mathrm{P} / \partial \ln \mathrm{L}=-5$.

Fig. 5 (a) The marginal stability boundaries of the $N=0$ symmetric ballooning modes in the equatorial $\left(\beta_{\|}, T_{\perp}, T_{\|}\right)$space for $\partial \ln P / \partial \ln L=-5,-2.5$, and -1 with the fixed parameters: $L$ $=6.6 \mathrm{RE}$ and azimuthal inode number $\mathrm{m}=50$. The $\tau=0$ curve is also shown. (b) The same stability boundaries expressed in the equatorial $\left(\alpha_{p}, \tau\right)$ space, where $\alpha_{p}$ is defined in Eq. (19).

Fig. 6 The field-aligned structure of (a) the three perturbed magnetric field components $\delta B_{\|}$, $\delta B_{\psi}$, and $-i \delta B_{\phi}$ normalized by $B_{o}$, and (d) the normalized electrostatic potential $\delta \Phi$ $\left(=\Phi / \omega L^{2} B_{0}\right.$, where $\Phi$ is the electrostatic potential) of a marginally stable $N=1$ antisymmetric ballooning mode for $L=6.6 R_{E}, T_{\perp} / T_{\|}(\theta=0)=1.6, \beta_{\|}(\theta=0)=1.179$ ( $\tau$ $=-0.1317$ ), azimuthal mode number $\mathrm{m}=50$, and $\partial \ln \mathrm{P} / \partial \ln \mathrm{L}=-2$. 
Fig. 7 The field-aligned structure of the three perturbed current density components (a) $-i \delta J_{\|}$, (b) $-\mathrm{i} \delta \mathrm{J}_{\psi},(\mathrm{c}) \delta \mathrm{J}_{\phi}$ normalized by $\left(\mathrm{B}_{0} / \mathrm{L}\right)$ of a marginally stable $\mathrm{N}=1$ antisymmetric ballooning mode with corresponding magnetic field perturbation shown in Fig. 6.

Fig. 8 (a) The marginal stability boundaries of the $\mathrm{N}=1$ antisymmetric mode in the equatorial $\left(\beta_{\|}, \mathrm{T}_{\perp} / \mathrm{T}_{\|}\right)$space for $\partial \ln \mathrm{P} / \partial \ln \mathrm{L}=-100,-50,-25,-10,-5,-2.5$, and -1 , respectively. with the fixed parameters: $L=6.6 R_{E}$ and azimuthal mode number $m=50$. The equatorial $\tau=0$ curve is also shown. (b) The same stability boundaries are shown in the equatorial $\left(\alpha_{p}, \tau\right)$ space. Note that for $\tau<0$, the $\alpha_{p}$ threshold is also negative and absolute values of $\alpha_{p}$ for $\tau<0$ are plotted.

Fig. 9 The field-aligned perturbed magnetic field structures of the marginally stable $N=1$ antisymmetric modes along a marginal stability curve in Fig. 7 (a) with equatorial values ol (a) $\mathrm{T}_{\perp} / \mathrm{T}_{\|}=1, \beta_{\|}=1.93(\tau=1)$, (b) $\mathrm{T}_{\perp} / \mathrm{T}_{\|}=1.3, \beta_{\|}=1.489(\tau=0.419)$, (c) $\mathrm{T}_{\perp} / \mathrm{T}_{\|}=$ 1.6, $\beta_{\|}=0.987(\tau=0.052)$, and (d) $T_{\perp} / T_{\|}=2, \beta_{\|}=0.575(\tau=-0.149)$. The fixed parameters are $\mathrm{L}=6.6 \mathrm{R}_{\mathrm{E}}$, azimuthal mode number $\mathrm{m}=5($ ), and $\partial \ln \mathrm{P} / \partial \ln \mathrm{L}=-5$. 


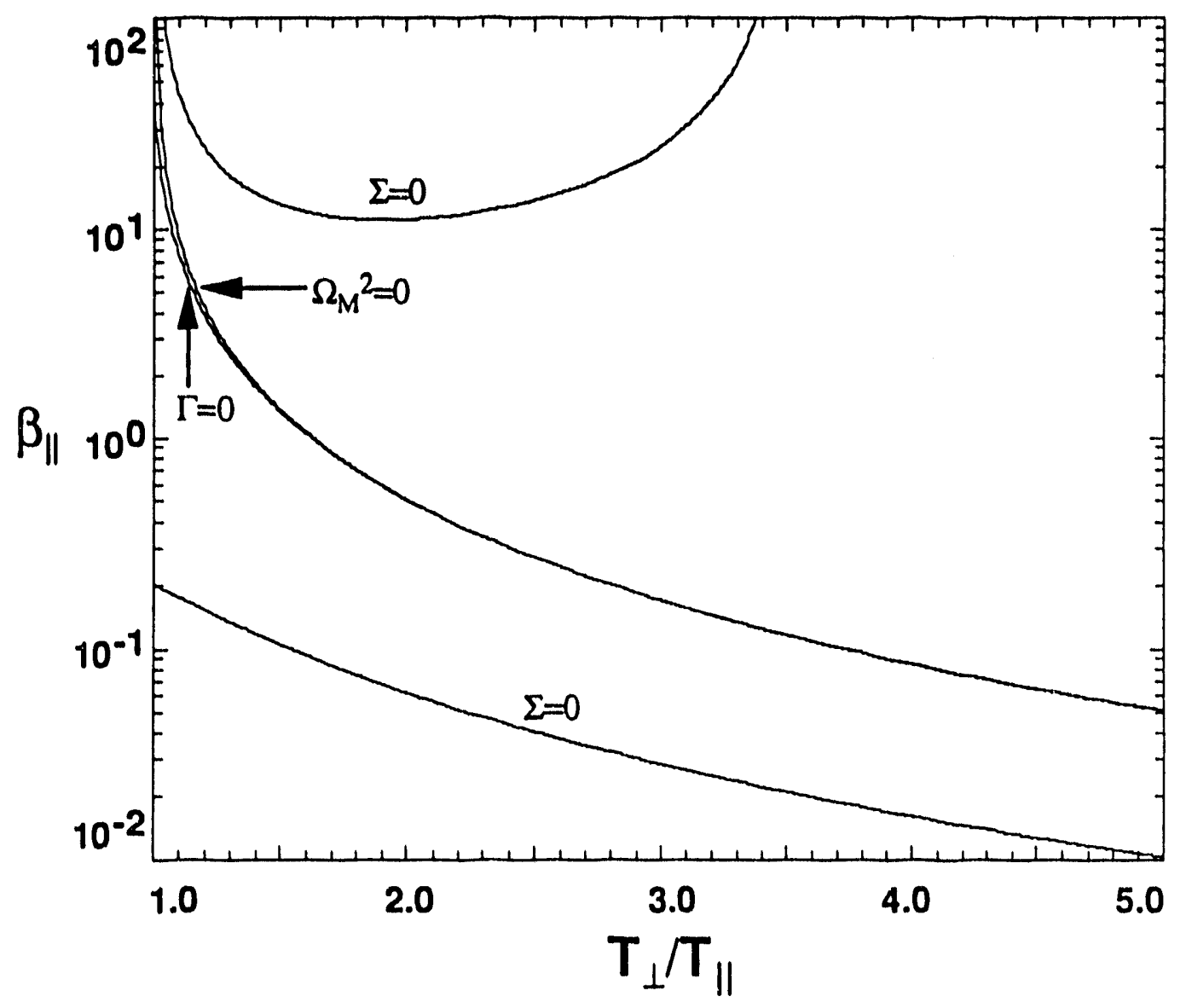

Fig. 1 

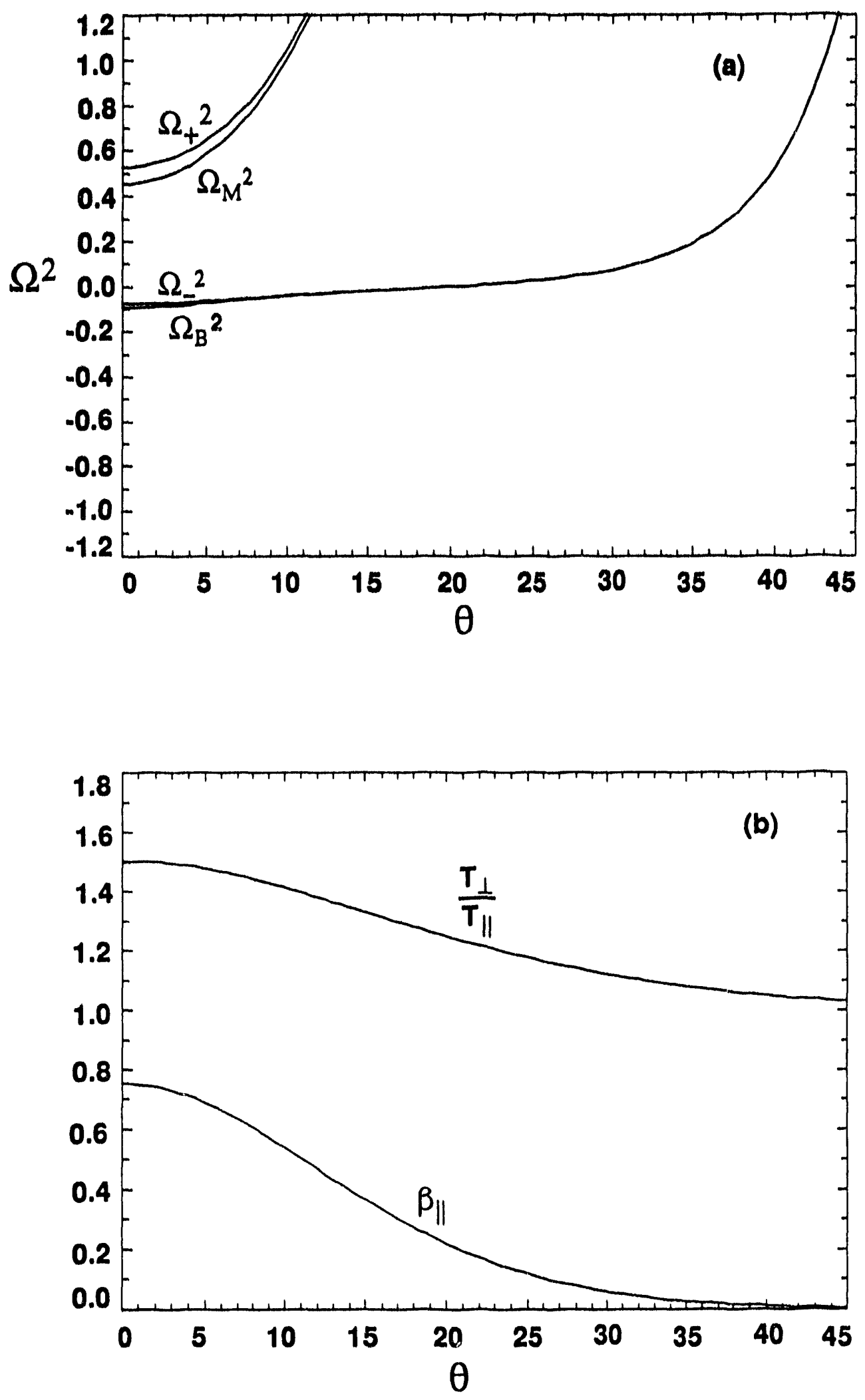

Fig. 2 

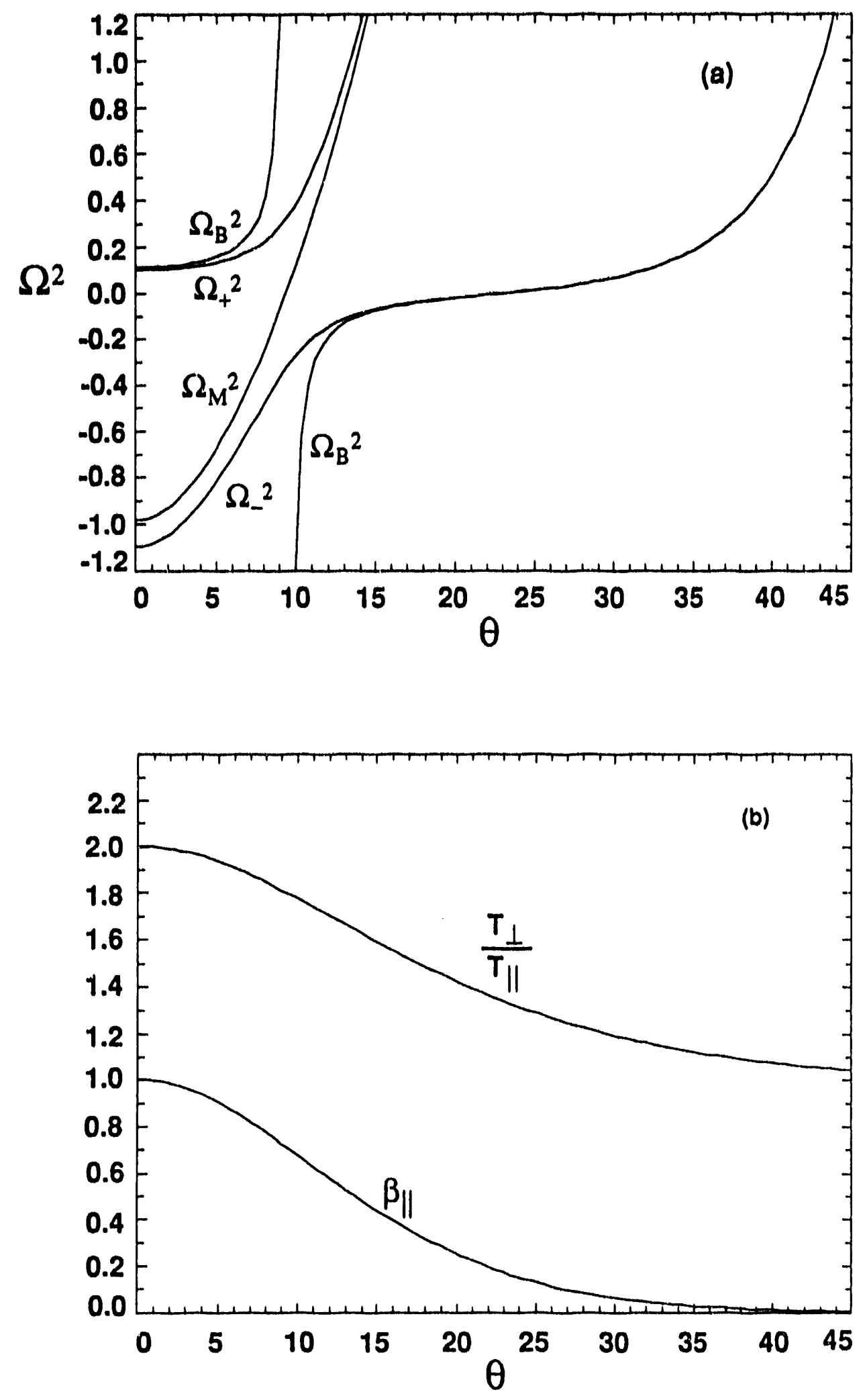

Fig. 3 

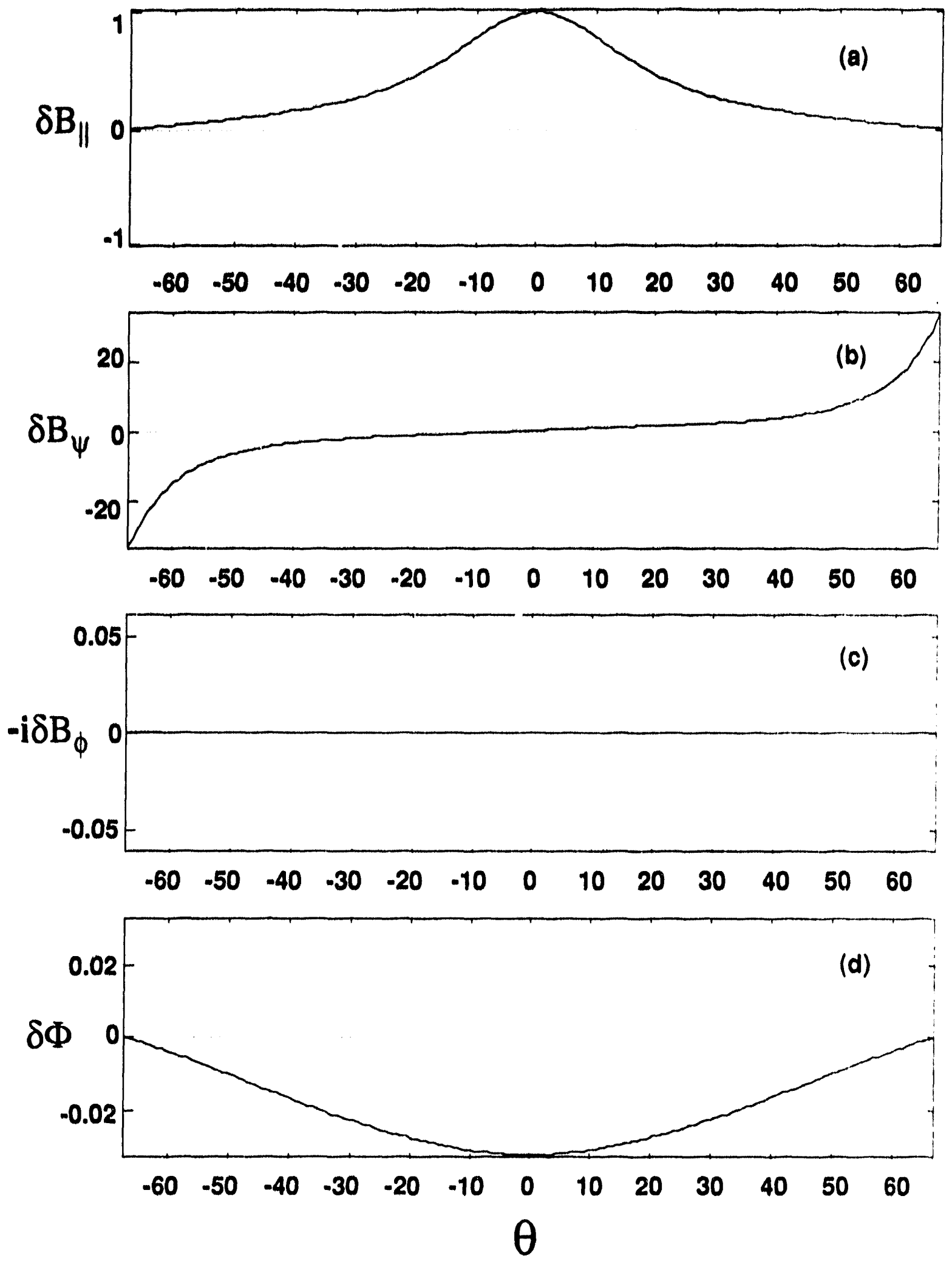

F1g. 4 

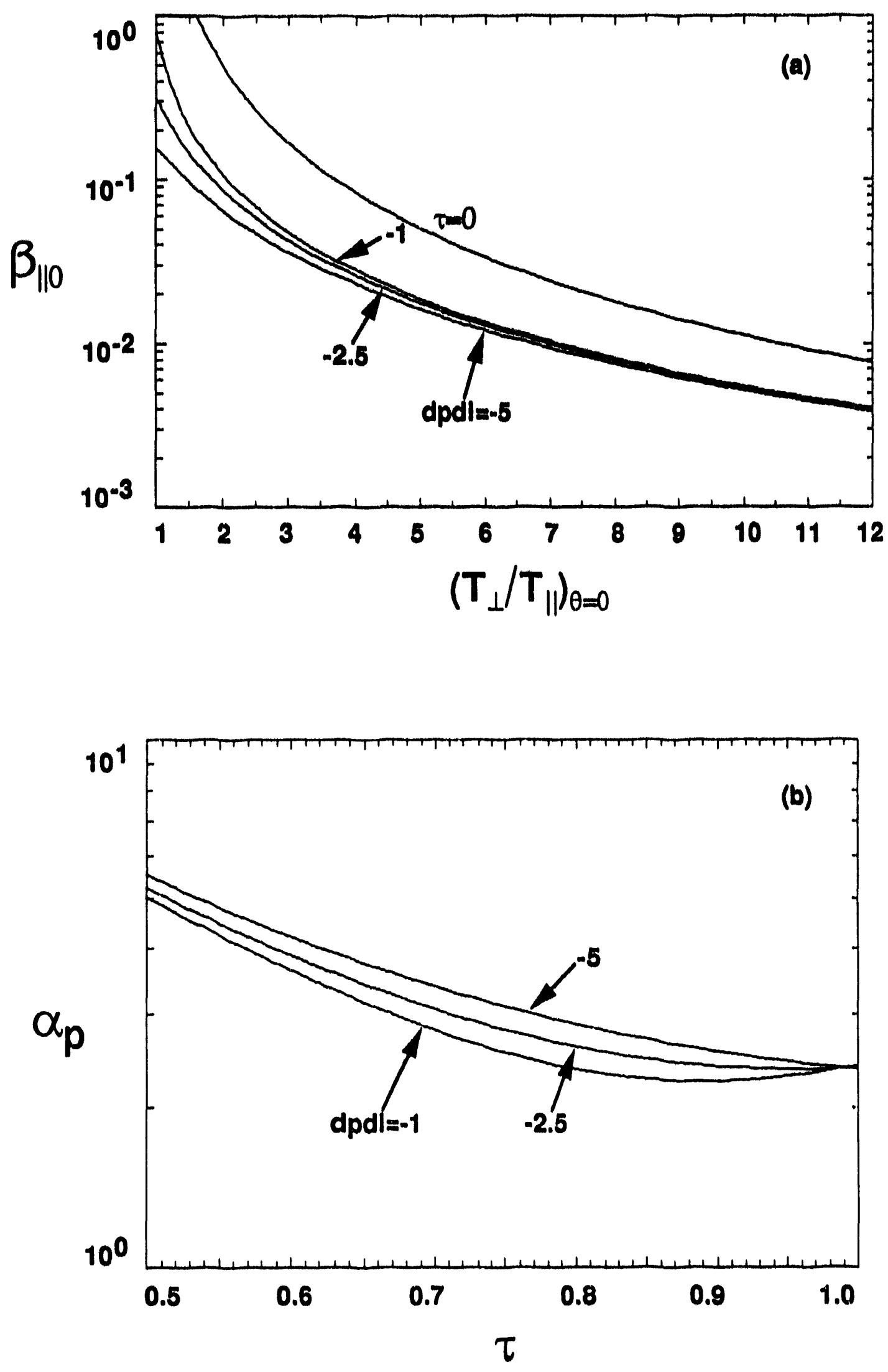

F1g. 5 

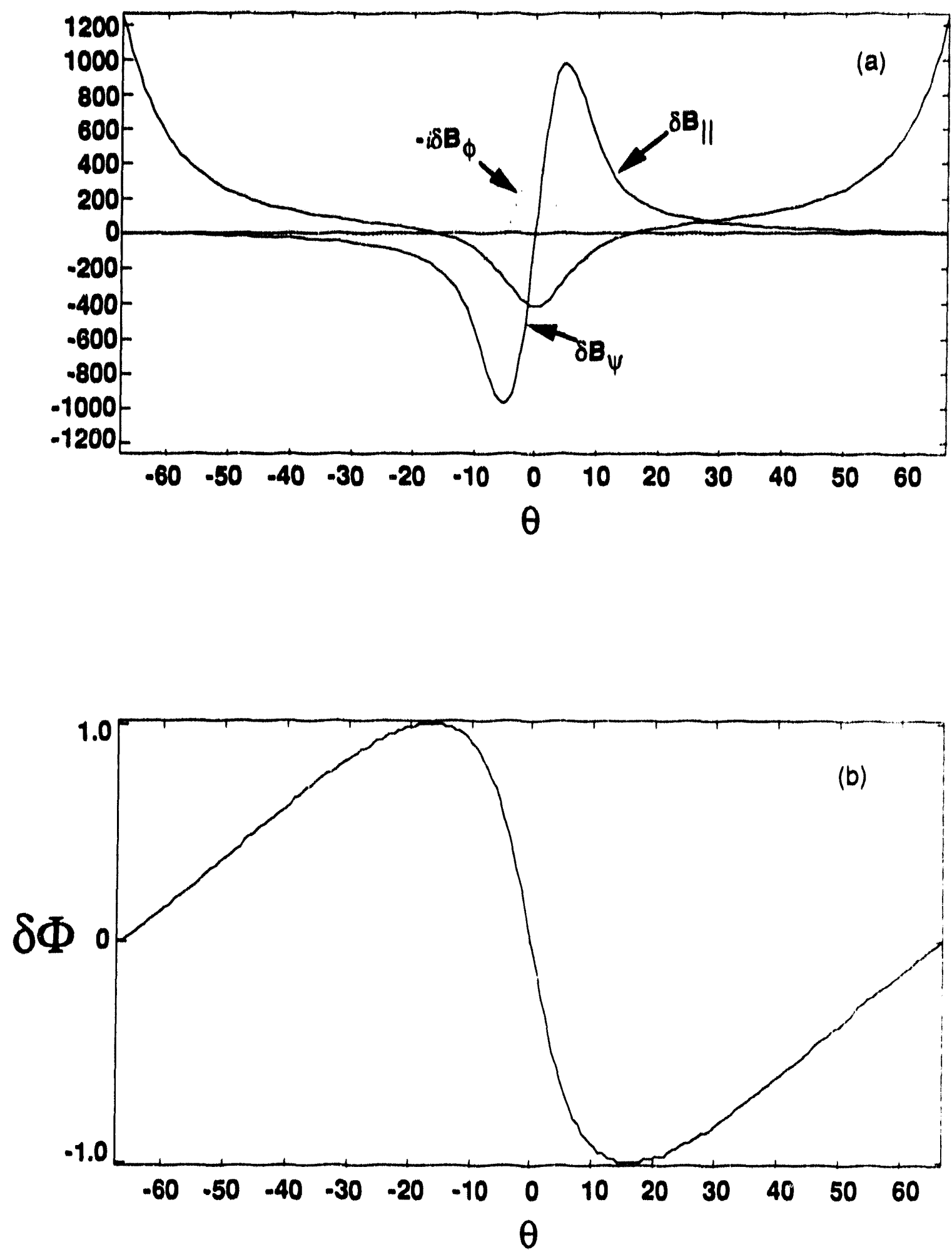

Fig. 6 

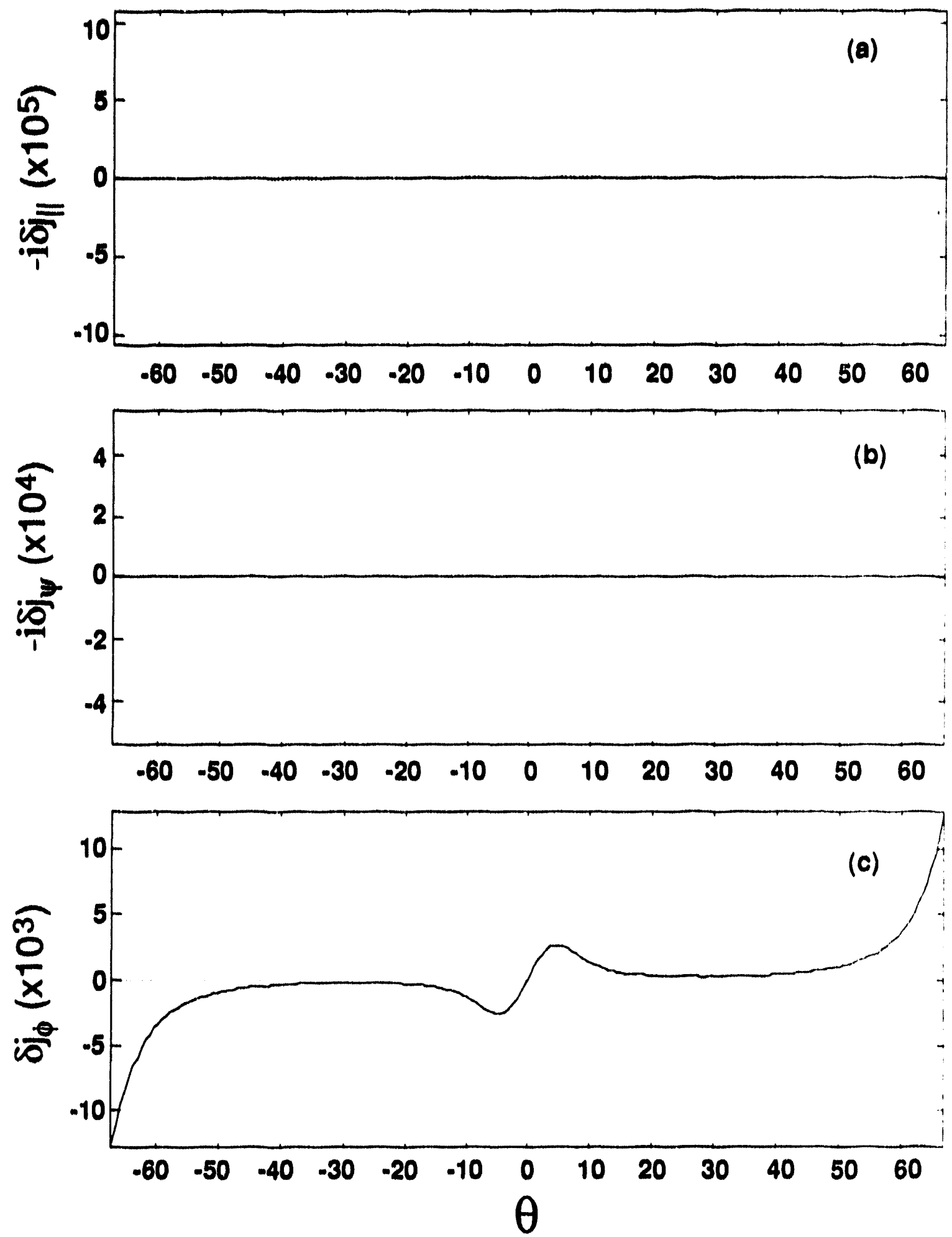

F1g. 7 

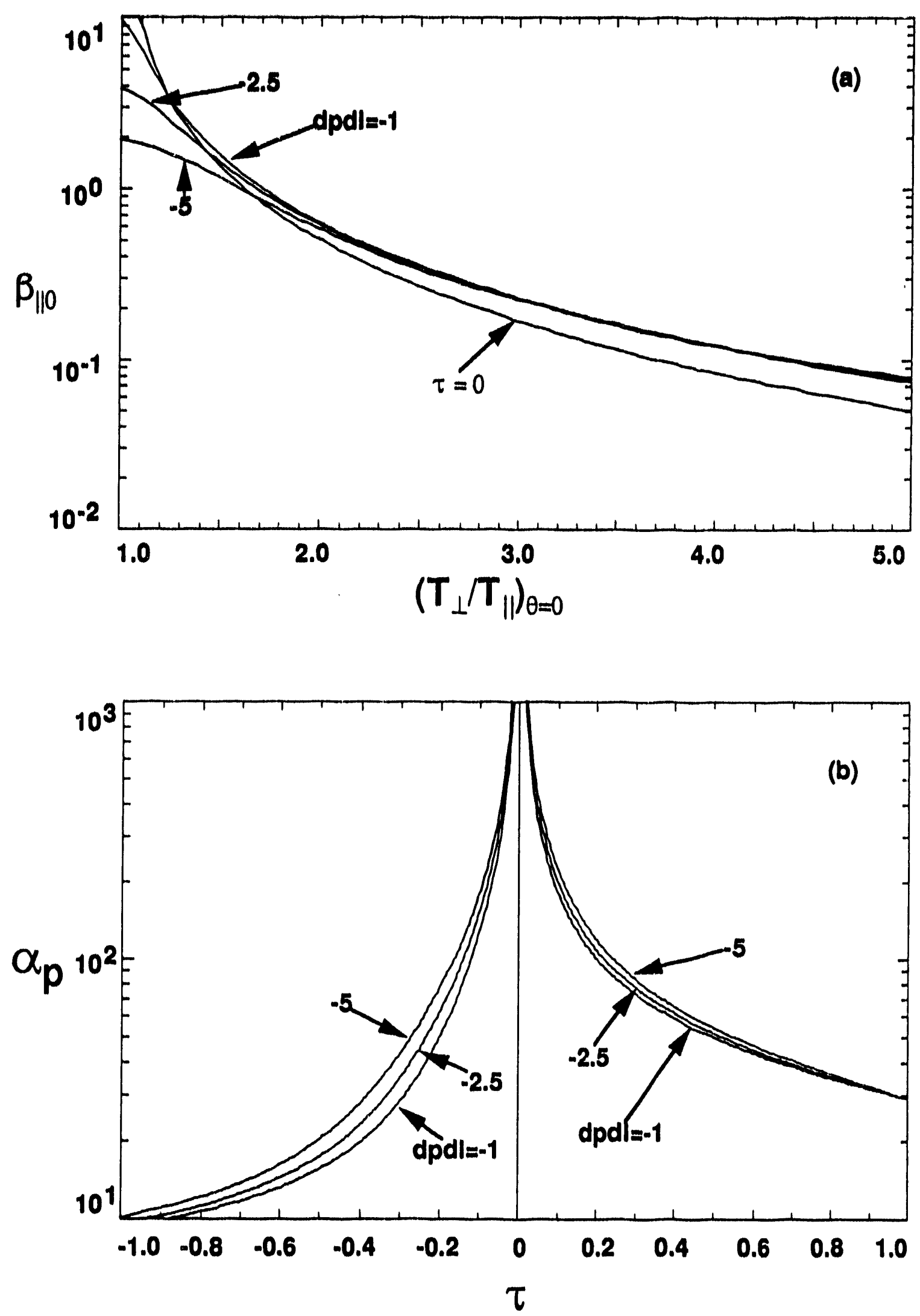

F1g. 8 

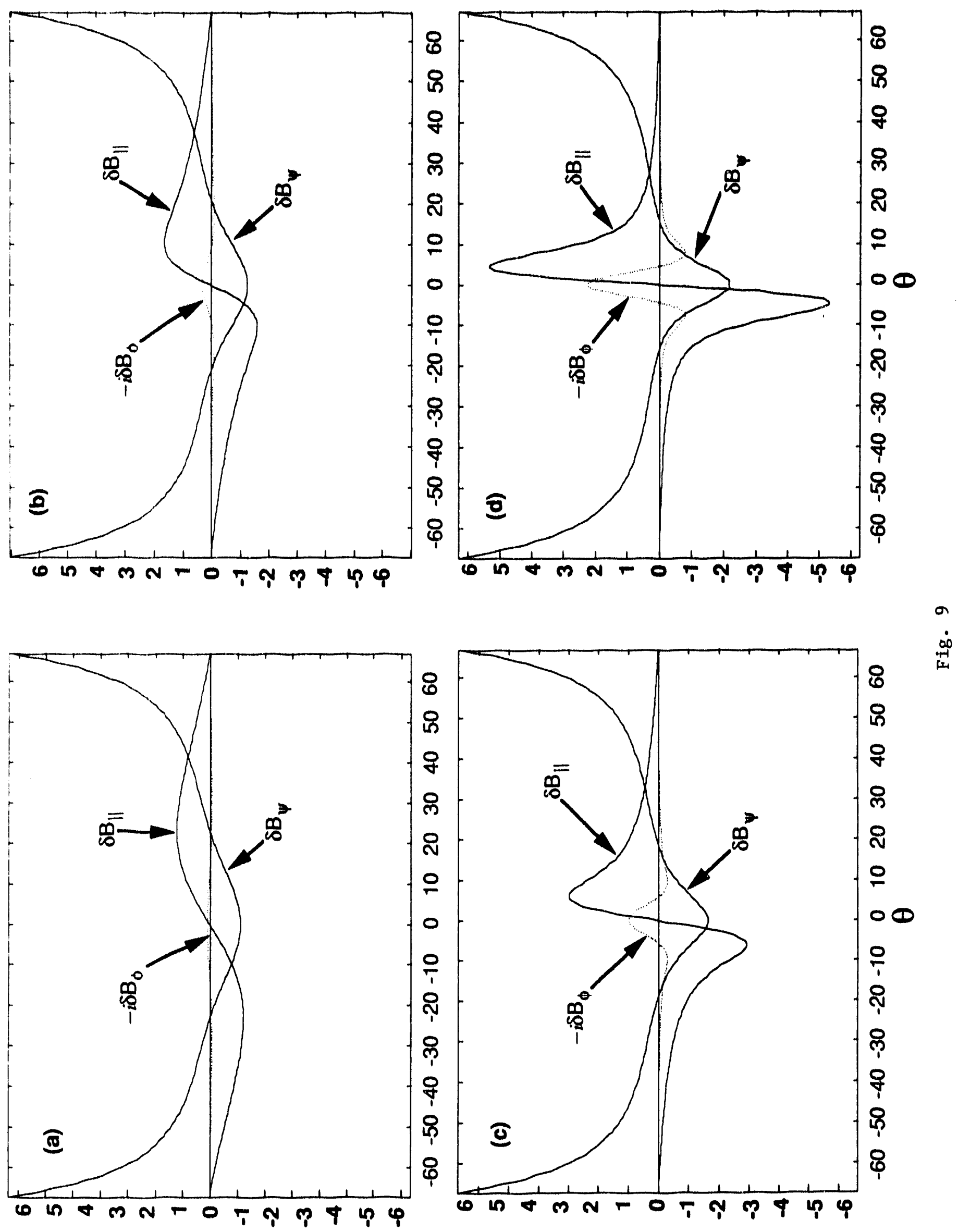
Dr. F. Peoloni, Univ. of Wollongong. AUSTRALIA

Prof. M.H. Brennan, Univ. of Sydney, AUSTRALIA

Plasma Rosearch Lab., Australian Nat. Univ., AUSTRALIA

Prof. I.R. Jones, Flinders Univ, AUSTRALIA

Prof. F. Cap, Inst for Theoretical Physics, AUSTRIA

Prof. M. Heindier, Institul for Theoretische Physik, AUSTRIA

Prof. M. Goossens, Astronomisch InstituUt, BELGIUM

Ecole Royalo Mitivire, Lab. de Phy. Plaemas, BELGIUM

Commission-Europeen, DG. XII-Fusion Prog., BELGIUM

Prof. R. Bouciqud, Rijksunivenibit Gent, BELGIUM

Dr. P.H. Sakanaka, Instituto Fiaica, BRAZIL

Inetitub Neciond Do Posquikas Especiair-INPE, BPUZIL

Documents Offico, Alomic Energy of Cenada Lid., CANADA

Dr. M.P. Bectynski, MPB Technologios, Inc., CANADA

Dr. H.M. Skaregard, Univ. of Seckatchowen, CANADA

Prof. J. Toichmann, Univ. of Montroal, CANADA

Prot. S.R. Sreenivasen, Univ. of Celgary, CANADA

Prof. T.W. Johnston, INRS-Energie, CANADA

Dr. R. Botton, Contre canadien de husion magnétique, CANADA

Dr. C.R. James, Univ. of Aborti, CANADA

Dr. P. Lukac, Komensketho Univorszita, CZECHOSLOVAKIA

The Librarian, Cutham Laboratory, ENGLAND

Library, R61, Ruthertord Appleton Laboratory, ENGLAND

Mrs. S.A. Hutchinson, JET Librery, ENGLAND

Dr. S.C. Shama, Univ. of South Pacific, FIJI ISLANDS

P. Mathonen, Univ. of Helsinki, FINLAND

Prol. M.N. Buseac, Ecole Polytechnique, FRANCE

C. Noutiot, Lab. de Physique dos Milioux lonises, FRANCE

J. Radat, CEN/CADARACHE - Bat 506, FRANCE

Prol. E. Economow, Univ. of Crele, GREECE

M5. C. Rinni, Univ. of loannina, GREECE

Dr. T. Mud, Academy Bibliographic Ser., HONG KONG

Proprint Library, Hungarian Acadomy of Sci., HUNGARY

Dr. B. DasGupta, Saha Inst. of Nuctoar Physics, INDIA

Dr. P. Kaw, Inst. for Plasma Research, INDIA

Dr. P. Rosenas, lsrael Inst of Tectinology, ISPAEL

Librarian, Intomational Contor for Theo Physics, ITALY

Miss C. De Palo, Associazione EURATOM-ENEA, ITALY

Dr. G. Grosso, Istituto di Fisica dol Plasma, ITALY

Prol. G. Rostangni, Istituto Gas lonizzati Del Cnr, ITALY

Dr. H. Yamato, Tochiba Res a Doved Centor, JAPAN
Prol. I. Kawakami, Hiroshima Univ., JAPAN

Prof. K. Nishikawa, Hiroshima Univ., JAPAN

Director, Japan Atomic Energy Research Inst., JAPAN

Prof. S. Itoh, Kyushu Univ., JAPAN

Research Into. Ctr., National Instit. for Fusion Science, JAPAN

Prot. S. Tenaka, Kyoto Univ., JAPAN

Library, Kyoto Univ., JAPAN

Prot. N. Inowe, Univ. of Tokyo, LAPAN

Secrotary, Plasme Section, Electrobechnical Lab., JAPAN

S. Mori, Tectmical Advisor, LAERI, JAPAN

Dr. O. Niterai, Kumamos Inst of Technology, JAPAN

J. Hyoon-Sook, Korea Alomic Energy Research InsL, KOREA

D.I. Choi, The Korea Adv. Inst of Sai. \& Tech., KOREA

Prol. B.S. Liby, Univ. of Waikato, NEW ZEALAND

Inst of Physics, Chinese Acad Sci PEOPLE'S REP. OF CHINA

Librey, Inst of Plasma Physics, PEOPLE'S REP. OF CHINA

Tringhua Univ. Library, PEOPLE'S REPUBLIC OF CHINA

Z Li, S.W. Inst Phycics, PEIJPLE'S REPUBLIC OF CHINA

Prof. J.A.C. Cebred, Institurio Superior Tecnico, PORTUGAL

Dr. O. Potrus, al I Cuza Univ., Pomania

Dr. J. de Villiers, Fusion Studies, AEC, S. AFRICA

Prof. M.A. Hellberg, Univ. of Natal, S. AFRICA

Prot. D.E. Kim, Pohang inst. of Sa. \& Tech., SO. KOREA

Prof. C.I.E.M.A.T, Fusion Division Library, SPAIN

Dr. L Sionflo, Univ. of UMEA, SWEDEN

Lorey, Royed Inst. of Tectnology, SWEDEN

Prof. H. Wihtemson, Chalmers Univ. of Tech., SWEDEN

Contro Phys. Des Plasmas, Ecole Polytech, SWITZERLAND

Bibliotheek, Inst. Voor Plasma-Fysica, THE NETHERLANDS

Asst. Prot. Dr. S. Cekir, Middo East Tech. Univ., TURKEY

D. V.A. Guktikh,Sci. Res. Inst. Electrophys.I Apparatus, USSR

Dr. D.D. Ayutov, Siberian Branch of Academy of Sa., USSR

D. G.A. Elicoov, I.V. Kurchatov Inst, USSR

Librarian, The Ukr.SSR Academy of Scionces, USSR

Dr. L.M. Kovrizhnykh, Inst. of General Physics, USSA

Kemforsctungsanlage GmbH, Zentralbibliothok, W. GERMANY

Bibliothek, Inst. For Plasmaforschung, W. GERMANY

Prof. K. Schinder, Ruhr-Universitat Bochum, W. GERMANY

Dr. F. Wagner, (ASDEX), Max-Planck-Institut, W. GERMANY

Librarian, Max-Plenck-Institut, W. GERMANY

Prof. R.K. Janev, Inst of Physics, YUgosLaVIA 

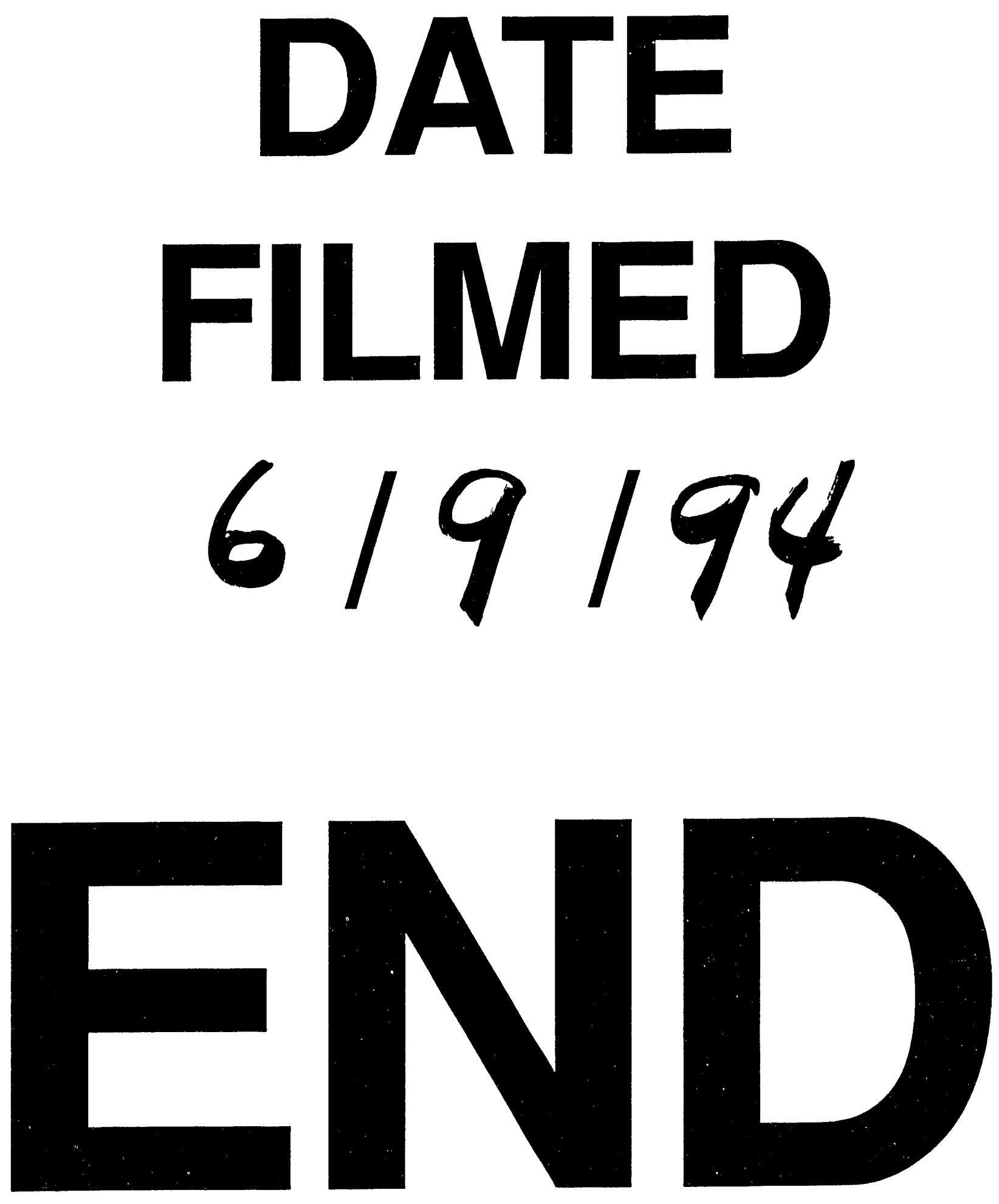
